University of Wollongong

Research Online

Faculty of Engineering and Information

Faculty of Engineering and Information

Sciences - Papers: Part B

Sciences

2019

\title{
Influence of molecular structure on lubrication of aqueous triblock copolymer lubricants between rutile surfaces: An MD approach
}

Thi D. Ta

University of Wollongong, thi@uow.edu.au

Anh Kiet Tieu

University of Wollongong, ktieu@uow.edu.au

Hongtao Zhu

University of Wollongong, hongtao@uow.edu.au

Shanhong Wan

University of Wollongong, shanhong@uow.edu.au

Hoang The Phan

University of Wollongong, htp342@uowmail.edu.au

See next page for additional authors

Follow this and additional works at: https://ro.uow.edu.au/eispapers1

Part of the Engineering Commons, and the Science and Technology Studies Commons

Research Online is the open access institutional repository for the University of Wollongong. For further information contact the UOW Library: research-pubs@uow.edu.au 


\title{
Influence of molecular structure on lubrication of aqueous triblock copolymer lubricants between rutile surfaces: An MD approach
}

\author{
Abstract \\ The influence of molecular structure on adsorption and lubrication of aqueous triblock copolymer on \\ TiO2surfaces has been investigated using a realistic MD model. Reference data obtained from DFT \\ calculations have been employed to develop an interaction potential between the copolymer and \\ TiO2surface. The results show that R Pluronics form an anchor-buoy-anchor structure absorbed on \\ TiO2surface and L Pluronic for buoy-anchor-buoy structure. The shear of tribo-system influences slightly \\ on the orientation of copolymers and non-slip behavior has been observed at solid-fluid interfaces due to \\ the strong adhesion strength of copolymer on rutile surface. The shear viscosity increases with \\ copolymer concentration and molecular chain length. L Pluronic has a lower shear stress than the R ones. \\ Disciplines \\ Engineering | Science and Technology Studies

\section{Publication Details} \\ Ta, T. D., Tieu, A. K., Zhu, H., Wan, S., Phan, H. T. \& Hao, J. (2019). Influence of molecular structure on \\ lubrication of aqueous triblock copolymer lubricants between rutile surfaces: An MD approach. Tribology \\ International, 130 170-183.

\section{Authors} \\ Thi D. Ta, Anh Kiet Tieu, Hongtao Zhu, Shanhong Wan, Hoang The Phan, and Jingcheng Hao
}




\title{
Influence of Molecular Structure on Lubrication of Aqueous Triblock Copolymer Lubricants between Rutile Surfaces: An MD Approach
}

\author{
T. D. Ta ${ }^{\mathrm{a}}$, A. K. Tieu ${ }^{\mathrm{a}}$, H. Zhu*a, S. Wan ${ }^{\mathrm{a}}$, H. T. Phan ${ }^{\mathrm{a}}$, and J. Hao ${ }^{\mathrm{b}}$

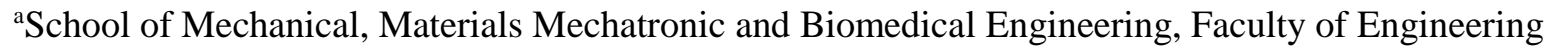 \\ and Information Sciences, University of Wollongong, Northfield Avenue, Wollongong, NSW, 2522, \\ Australia
}

${ }^{\mathrm{b}}$ School of Chemistry and Chemical Engineering \& Key Laboratory of Colloid and Interface Chemistry, Shandong University, Jinan, 250100, PR China Electronic 


\begin{abstract}
The influence of molecular structure on adsorption and lubrication of aqueous triblock copolymer on $\mathrm{TiO}_{2}$ surfaces has been investigated using a realistic MD model. Reference data obtained from DFT calculations have been employed to develop an interaction potential between the copolymer and $\mathrm{TiO}_{2}$ surface. The results show that $\mathrm{R}$ Pluronics form an anchor-buoy-anchor structure absorbed on $\mathrm{TiO}_{2}$ surface and L Pluronic for buoy-anchor-buoy structure. The shear of tribo-system influences slightly on the orientation of copolymers and non-slip behavior has been observed at solid-fluid interfaces due to the strong adhesion strength of copolymer on rutile surface. The shear viscosity increases with copolymer concentration and molecular chain length. L Pluronic has a lower shear stress than the $\mathrm{R}$ ones.
\end{abstract}

KEYWORDS: adsorption; aqueous lubricant; triblock copolymer; molecular dynamics. 


\section{INTRODUCTION}

Aqueous Pluronic triblock copolymer lubricants have been applied as the aqueous metalworking lubricants due to their effective tribological performance and environmental friendly properties $[1,2]$. There are two types of Pluronic classified as L and R series depending on the molecular structure of polyethyleneoxide (PEO) and polypropyleneoxide (PPO) blocks. The normal (L) copolymers are characterized by a central non-polar PPO block and two ending polar PEO blocks with the following structure: $\mathrm{PEO}_{n}-\mathrm{PPO}_{\mathrm{m}}-\mathrm{PEO}_{\mathrm{n}}[3]$. In contrast, the R series are characterized by the molecular structure: $\mathrm{PPO}_{\mathrm{n}}-\mathrm{PEO}_{\mathrm{m}}-\mathrm{PPO}_{\mathrm{n}}$ [4]. Both $\mathrm{L}$ and $\mathrm{R}$ copolymers dissolve well in water due to the amphiphilic PEO block, whilst PPO is less soluble due to its hydrophobic property [5]. Experimental studies have revealed that the hydrophilic property of PEO block is characterized by a strong hydrogen bond between its ether oxygen with hydrogens of water, whilst this hydrogen bond is lower for PPO block [6, 7]. The binding energy of 1,2-DME, a representative model of PEO block to water is comparable to waterwater binding, indicating a strong hydrogen bonding between PEO and water [8]. However, this hydrogen bond depends strongly on the concentration and temperature of the solution [9-11]. The copolymers could exist as isolated chains or unimers at low temperature and/or low concentration, micelles at high copolymer concentration and/or temperature, and dual-phase at intermediate state [9].

Due to the difference in chemical and structural properties of constituent blocks, the adsorption of triblock copolymer in aqueous solution is an interesting phenomenon. The buoy-anchor-buoy (B-AB) or anchor-buoy-anchor (A-B-A) structures of adsorbed copolymer depend on the relative interaction between PEO/PPO blocks and the surface [12]. For hydrophobic surfaces, the investigations of adsorption of triblock copolymers on hydrophobic organic surfaces such as polystyrene latex [11], polyethylene and polypropylene $[13,14]$, show that the hydrophobic interactions are driven by PPO chains, whilst the PEO chains extend into the bulk aqueous solution and form a steric bulky layer [1517]. For hydrophilic surfaces, such as cellulose, the adsorption of triblock copolymers takes place predominantly through hydrophilic PEO blocks [14]. Although comprehensive investigations on the physisorption of $\mathrm{L}$ and $\mathrm{R}$ Pluronics have been carried out, their adsorption energy has yet to be determined.

The mineral and amorphous polymeric surfaces are usually applied to investigate the influence of the surface's hydrophobicity on architectural properties of triblock copolymers [11, 13, 14, 18]. However, an insight into the adsorption of triblock copolymers on metal and metal oxide surfaces at the atomic level is still unclear, despite their wide application in metal forming $[1,15,19,20]$. Although experimental investigations of copolymer have made a significant progress for a few decades, numerical studies have just been developed over a shorter time due to limitations in numerical development and the expensive computation. As copolymers are the large molecules, the simulations of Pluronic 
copolymers are usually limited to mesoscopic dynamics [21, 22]. Many other efforts have been dedicated to shed light on the complex molecular structure of polymers at the liquid-solid interfaces using an approach from an atomic scale to mesoscale [23, 24]. The Hamaker theory [25], which simplifies the van der Waals ( $\mathrm{vdW}$ ) interaction by pairwise summation of the individual contributions over the volumes of interacting bodies, has been used to describe the interaction between the polymers as well as that between the polymers and solid surface [26]. This simplified vdW treatment, however, only works effectively for homogeneous material properties and it shows a discrepancy for thin lubricant film [26]. Although the simulation time and model's domain have been improved significantly by using these approaches, many important chemical properties such as hydrogen bonding, local molecular conformations, electrostatic interactions, and crystalline surfaces structures are usually excluded. Moreover, the relative adhesive strengths between the PPO/PEO beads and the surface are usually chosen such that the adhesive strength of the PPO bead is stronger than that from PEO to mimic the hydrophobic surface [27].

For MD simulation, the potentials for individual PEO and PPO blocks of copolymer and copolymerwater system have been derived by Smith and colleagues [5, 8, 28-32]; however the force field (FF) that satisfactorily describes the interactions between copolymer and a particular metal or metal oxide surface is still limited. In an effort to investigate the adsorption of $\mathrm{PEO}$ on $\mathrm{TiO}_{2}$ surface, the interfacial FF between PEO block and $\mathrm{TiO}_{2}$ surface has been derived from quantum chemistry calculation [33]. However, this FF was carried out from a cluster of short dimethyl ether (DME)- $\mathrm{TiO}_{5} \mathrm{H}_{9}$, and it is not suitable for a system of triblock copolymer adsorbed on $\mathrm{TiO}_{2}$ surface. Recently, an interfacial FF using united-atom model has been successfully derived by the authors based on ab initio data for system of reverse (R) triblock copolymers on iron surface [34]. A new potential has been developed in this work to describe the interfacial interactions between PEO and PPO with rutile surface using ab initio calculations.

This work, therefore, investigates the effect of molecular structure on the adsorption and tribological performance of both $\mathrm{L}$ and $\mathrm{R}$ Pluronics in aqueous lubricant confined between $\mathrm{TiO}_{2}$ surfaces. Additionally, this theoretical investigation also aims to support previous experimental studies $[1,15$, 19, 20, 35]. An MD model has been developed to simulate realistically the self-assembled molecular structure of adsorbed Pluronics at the atomic scale. A new potential was developed using density functional theory (DFT) data to present the interactions between Pluronics and the $\mathrm{TiO}_{2}$. The absorbed molecular architectures of Pluronics, along with their adsorption energies and rheological properties, have been analyzed in this study. There are many factors such as charge and roughness that affect the adhesion energy of lubricant on the surface and between counter surfaces [36]. As a first part in a series of work regarding this topic, an ideal smooth surface was employed in this work to investigate the effect of molecular structure. The influences of surface properties will be published in the near future. 


\section{SIMULATION METHODOLOGY}

\subsection{Tribo-systems}

The tribo-system of $\mathrm{TiO}_{2}$ surfaces lubricated by aqueous copolymer lubricant in Fig. 1 is periodic in lateral directions and has the simulation domain of $120 \times 120 \times 311 \AA^{3}$. A thickness of $10 \AA$ has been set for the surface, which is comprised of 20280 atoms. The rigid $(1,3)$ and thermostat layers $(2,4)$ were applied to the surface to constraint and control the temperature, respectively. The solution was initially prepared by assigning a thin layer of copolymer molecules (Table 1) sandwiched between water layers.

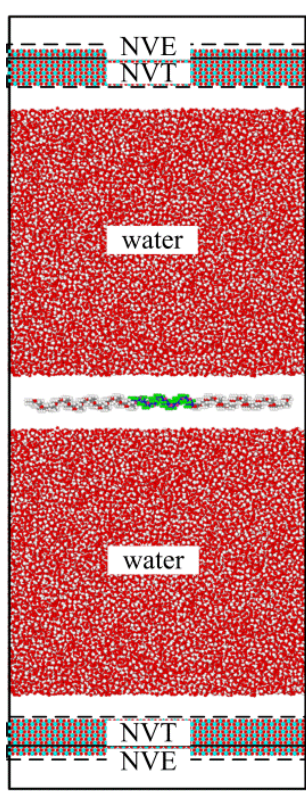

Z Initial

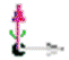

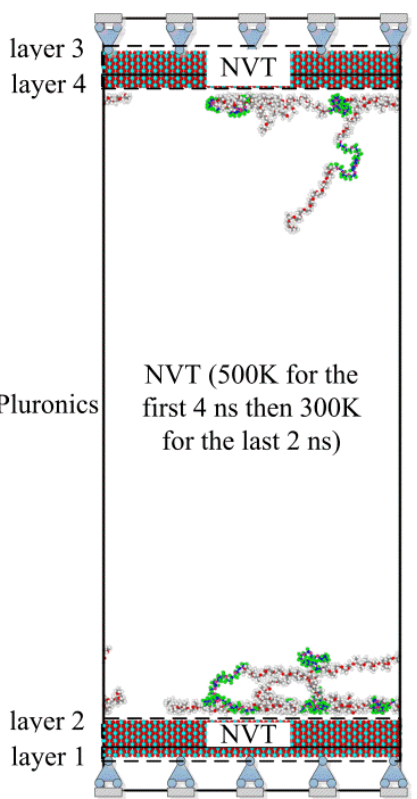

(I) Relaxation

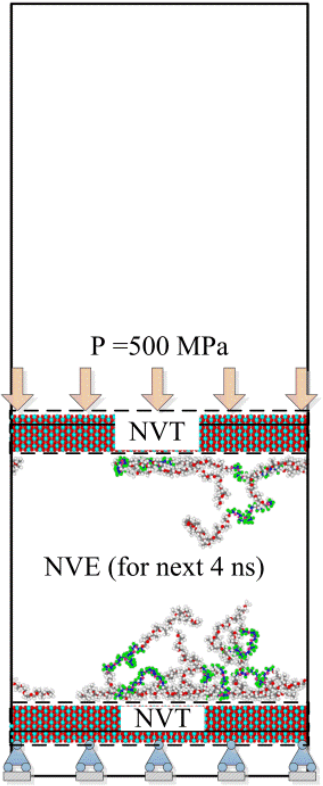

(II) Compression

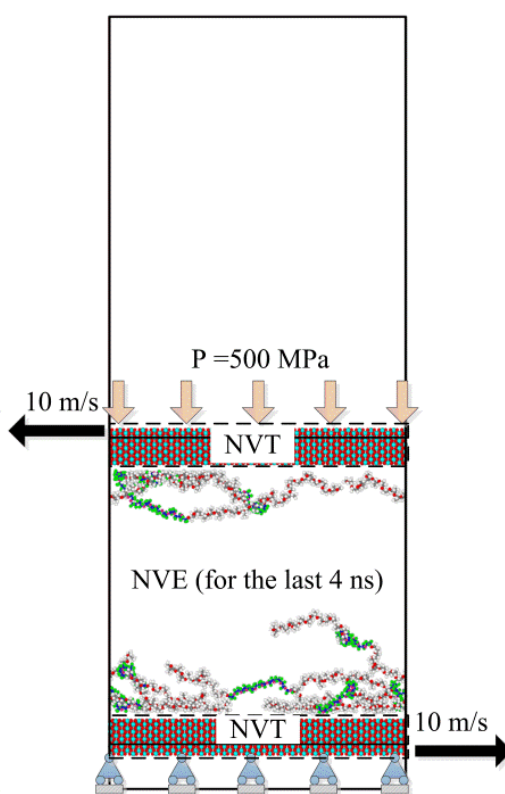

(III) Confined shear

Fig. 1. Aqueous Pluronic copolymer lubricant confined between $\mathrm{TiO}_{2}(001)$ surfaces subjected to an applied pressure of $500 \mathrm{MPa}$ at $300 \mathrm{~K}$. Water is not presented in subsequent figures. Cyan and red colors present $\mathrm{Ti}$ and $\mathrm{O}$ in the surface, respectively. $\mathrm{C}, \mathrm{H}$, and $\mathrm{O}$ atoms are presented respectively by grey, white, and red colors in PPO block and water, while the corresponding colors of blue, green, and pink are for PEO block.

The simulation, which was similar to previous research by the authors, includes three stages $[34,37$, 38]. (I) Firstly, the rigid layers were fixed and an NVE ensemble was applied to them, while the remains of surface were free and thermostated at $300 \mathrm{~K}$ by Nose-Hoover NVT ensemble. These settings were set for all simulation stages. A temperature of $500 \mathrm{~K}$ has been applied to the lubricant during the first 4 ns to increase the mobility of the long copolymer chains, while the temperature of $300 \mathrm{~K}$ was applied for the last 2 ns of this relaxation stage. Since Pluronics are large molecules which have a number of 
local minima in energy, an annealing temperature at $500 \mathrm{~K}$ is therefore necessary to obtain an optimal equilibrium state $[34,39]$. (II) In the second stage, the system was gradually compressed by applying a pressure of $500 \mathrm{MPa}$ on the rigid layer (layer 3) of upper wall for $1.0 \mathrm{~ns}$. A movement limit of 0.001 $\AA$ per timestep has been applied to avoid highly overlapped atoms under a sudden impact of applied load. This load was then applied for another $3 \mathrm{~ns}$. In this stage, the upper rigid atoms were fixed in the lateral directions, whilst they were allowed to move in the $\mathrm{Z}$ direction. Meanwhile, the lower rigid atoms were constrained in all directions. (III) In the last simulation stage, the normal load and shearing velocity of $10 \mathrm{~m} / \mathrm{s}$ were applied to the rigid layers. The simulation time in each stage was chosen to achieve the steady state of interaction energy between the copolymer and the surface.

Table 1

Molecular formula, weight percentage of PEO block in copolymer molecule, weight percentage of copolymer in water solution, number of copolymers and $\mathrm{H}_{2} \mathrm{O}$ for different atomic mass concentrations in different Pluronics.

\begin{tabular}{|c|c|c|c|c|c|}
\hline Pluronic & Molecular formula & $\begin{array}{l}\text { PEO } \\
\% \text { wt }\end{array}$ & copolymer wt $\%$ & no. copolymers & no. water \\
\hline \multirow{2}{*}{ 17R2 } & \multirow{2}{*}{$\mathrm{PPO}_{15}-\mathrm{PEO}_{10}-\mathrm{PPO}_{15}$} & \multirow{2}{*}{20} & 2.1 & 10 & 55420 \\
\hline & & & 16.0 & 76 & 47446 \\
\hline \multirow{2}{*}{ 17R4 } & \multirow{2}{*}{$\mathrm{PPO}_{14}-\mathrm{PEO}_{24}-\mathrm{PPO}_{14}$} & \multirow{2}{*}{40} & 2.3 & 8 & 55300 \\
\hline & & & 16.1 & 56 & 47520 \\
\hline \multirow{2}{*}{$25 \mathrm{R} 2$} & \multirow{2}{*}{$\mathrm{PPO}_{21}-\mathrm{PEO}_{14}-\mathrm{PPO}_{21}$} & \multirow{2}{*}{20} & 2.0 & 6 & 55560 \\
\hline & & & 15.9 & 50 & 47700 \\
\hline \multirow{2}{*}{ L62 } & \multirow{2}{*}{$\mathrm{PEO}_{6}-\mathrm{PPO}_{32}-\mathrm{PEO}_{6}$} & \multirow{2}{*}{20} & 1.9 & 8 & 55560 \\
\hline & & & 16.2 & 68 & 47440 \\
\hline
\end{tabular}

A low concentration of around $2 \%$ has been used to observe the molecular structure of copolymers on the surface $[15,20]$. Meanwhile, a higher concentration of triblock copolymer of around $16 \%$ has been used to consider the influence of concentration on adsorption and rheological properties of tribosystems [27, 34]. This high concentration was chosen as the experimental results revealed that the adsorbed copolymer at the solid-fluid interface was more condense than from the bulk stage $[15,20]$. Therefore, a higher concentration has been considered when the behavior of triblock copolymers was simulated on a solid surface at the atomic scale $[27,34]$. The simulation procedure for this case was similar to that which was used at low concentration.

\subsection{Force Fields}

The SPC/E potential was applied for $\mathrm{H}_{2} \mathrm{O}$ [40], while the classical FF with all-atom model was applied for triblock copolymer [41]. The intra- and intermolecular interactions of Pluronics such as bonding, 
bending, and dihedral of polymer were considered in this simulation. The Matsui-Akaogi model was applied for rutile surfaces [42]. This model has been used widely in $\mathrm{MD}$ simulations of $\mathrm{TiO}_{2}$ systems $[43,44]$. The Buckingham potential obtained from Bandura and Kubicki's model has been used to describe the nonbond interactions between $\mathrm{TiO}_{2}$ surface and $\mathrm{H}_{2} \mathrm{O}$ [43]. The FF for PEO-water interaction, which has been developed upon the quantum chemistry calculations of DME interacted with water, was applied for interactions between copolymer and water [8]. This FF is also applicable for PPO and shows a good agreement with quantum chemistry calculation of DMP-water system [31]. The new FF that describes the interactions between the copolymer and $\mathrm{TiO}_{2}$ surface was derived in this work and described in following section. The bond and angle of water molecule were constrained using the SHAKE algorithm [45].

The vdW and the Coulombic interactions were truncated by a cutoff distance of $9.5 \AA$, which has been chosen after a careful checking of the potential for each pairwise interaction. Particularly, the energy curves for different pairwise interactions are convergence to zero when $\mathrm{r}_{\mathrm{ij}}>5 \AA$. This cutoff distance was therefore chosen in this work to minimize the error for Lennard-Jones (LJ) 12-6 potential. The truncation of the vdW potential is usually chosen at distance of $3 \sigma_{\mathrm{ij}}\left(\sigma_{\mathrm{ij}}\right.$ is the equilibrium distance between a pair of atoms $i$ and $j$ ) in MD simulation. In the fitted force field shown in the following result section, the $\sigma_{\mathrm{ij}}$ values are $<3.2 \AA$ (except Ti-H pair which has $\varepsilon \sim 0$ ). When this rule was applied, the maximum cutoff distance of $9.5 \AA$ was obtained and has been applied throughout of this work.

A particle-particle particle-mesh (PPPM) solver method was applied to correct the long range Coulombic interactions [46]. The numerical integration of atomic classical equations of motion was implemented using Verlet algorithm with a time step of $2.0 \mathrm{fs}$. The atomic charges for PPO block in copolymers and 1,2-DMP molecule were obtained from previous quantum calculations by Smith et al. [31], whilst the charges for PEO block and 1,2-DME molecules were derived from the work by Borodin et al. [28]. These electrostatic models were used as they were derived from second-order perturbation theory (MP2) that predicts more accurately the results for hafnocenes than B3LYP which is commonly used in DFT calculations [47].

Although an advanced reactive $\mathrm{FF}$ has been derived for the system of $\mathrm{Ti} / \mathrm{O} / \mathrm{C} / \mathrm{H}$ and applied to investigate the interactions between $\mathrm{TiO}_{2}$ nanoparticles and water with $\mathrm{Na}^{+}$and $\mathrm{Cl}^{-}$, methanol, and formic acid [48, 49], its high computational cost and a much smaller time step have limited the system to a few thousand atoms only. Although this domain is significantly larger than that can be handled by the DFT calculation, it is still very small compared to the current system. The traditional FF is therefore an optimal choice for the chosen domain of the current system. 


\subsection{Force field development for $\mathrm{PEO} / \mathrm{PPO}-\mathrm{TiO}_{2}$ interactions}

The adsorption energies $\left(E_{a d s}\right)$ along with atom's Cartesian coordinates gained from quantum calculations have been used as the training set. A FF parameterization using a generalized pattern searches algorithm has been applied to derive the LJ 12-6 FF parameters for the interactions between the copolymer and rutile surface [50]. The non-bond interactions were given in equation 1 :

$$
E_{i j}=\sum_{\mathrm{i}, \mathrm{j}} 4 \epsilon_{\mathrm{ij}}\left[\left(\sigma_{\mathrm{ij}}^{o} / r_{\mathrm{ij}}\right)^{12}-\left(\sigma_{\mathrm{ij}}^{o} / r_{\mathrm{ij}}\right)^{6}\right]+\sum_{\mathrm{i}, \mathrm{j}} q_{\mathrm{i}} q_{\mathrm{j}} / r_{\mathrm{ij}},
$$

where $i j=\mathrm{Ti}-\mathrm{O}, \mathrm{Ti}-\mathrm{C}, \mathrm{Ti}-\mathrm{H}, \mathrm{O}_{\mathrm{Ti}}-\mathrm{O}, \mathrm{O}_{\mathrm{Ti}}-\mathrm{C}$, and $\mathrm{O}_{\mathrm{Ti}}-\mathrm{H}$. There were twelve unknown nonbond $\mathrm{FF}$ parameters available to fit including six parameters of the depth of the potential well $\left(\epsilon_{\mathrm{Ti}-\mathrm{O}}\right.$, $\left.\epsilon_{\mathrm{Ti}-\mathrm{C}}, \epsilon_{\mathrm{Ti}-\mathrm{H}}, \epsilon_{\mathrm{O}_{\mathrm{Ti}}-\mathrm{O}}, \epsilon_{\mathrm{O}_{\mathrm{Ti}}-\mathrm{C}}, \epsilon_{\mathrm{O}_{\mathrm{Ti}}-\mathrm{H}}\right)$ and another six parameters of finite distances at which the interparticle potential was zero $\left(\sigma_{\mathrm{Ti}-\mathrm{O}}, \sigma_{\mathrm{Ti}-\mathrm{C}}, \sigma_{\mathrm{Ti}-\mathrm{H}}, \sigma_{\mathrm{O}_{\mathrm{Ti}}-\mathrm{O}}, \sigma_{\mathrm{O}_{\mathrm{Ti}}-\mathrm{C}}, \sigma_{\mathrm{O}_{\mathrm{Ti}}-\mathrm{H}}\right)$. As presented in Fig. 2 , an inhouse code has been written in Matlab to fit these parameters. The Coulombic energy $\left(E_{\text {Coul }}\right)$ was evaluated in LAMMPS using the atomic partial charges obtained from quantum calculations. The remaining vdW energy $\left(E_{L J}\right)$ in the following equation was used as the reference data for the current fitting.

$$
E_{L J}=E_{a d s}-E_{\text {Coul }}
$$

In this fitting approach, the minimum cost function $f\left(\varepsilon_{\mathrm{i}}, \sigma_{\mathrm{i}}\right)$, defined as:

$$
f\left(\varepsilon_{\mathrm{i}}, \sigma_{\mathrm{i}}\right)=\sqrt{\frac{\sum_{i} \omega_{i} \Delta E_{i}}{\sum_{i} \omega_{i}}}
$$

where $\omega_{i}$ was the weighting factor of configuration $i$, while $\Delta E_{i}$ was the percentage of energy difference between the evaluated LJ 12-6 potential $\left(E_{i}\left(\varepsilon_{\mathrm{i}}, \sigma_{\mathrm{i}}, r_{i}\right)\right)$ and the vdW energy obtained from DFTD calculation $\left(E_{i}^{D F T D}\right)$. It is expressed by following equation:

$$
\Delta E_{i}=\frac{E_{i}\left(\varepsilon_{\mathrm{i}}, \sigma_{\mathrm{i}}, r_{i}\right)-E_{i}^{D F T D}}{E_{i}\left(\varepsilon_{\mathrm{i}}, \sigma_{\mathrm{i}}, r_{i}\right)} .100 \%
$$

The initial parameters for $\mathrm{Ti}-\mathrm{O}, \mathrm{Ti}-\mathrm{C}$, and $\mathrm{Ti}-\mathrm{H}$ pairwise were directly taken from the work by Borodin et al. [33], whilst the initial parameters for $\mathrm{O}_{\mathrm{Ti}}-\mathrm{O}, \mathrm{O}_{\mathrm{Ti}}-\mathrm{C}$, and $\mathrm{O}_{\mathrm{Ti}}-\mathrm{H}$ pairwise were refitted from Buckingham potential obtained from their work as well. The fitting procedure is illustrated in Fig. 2. 


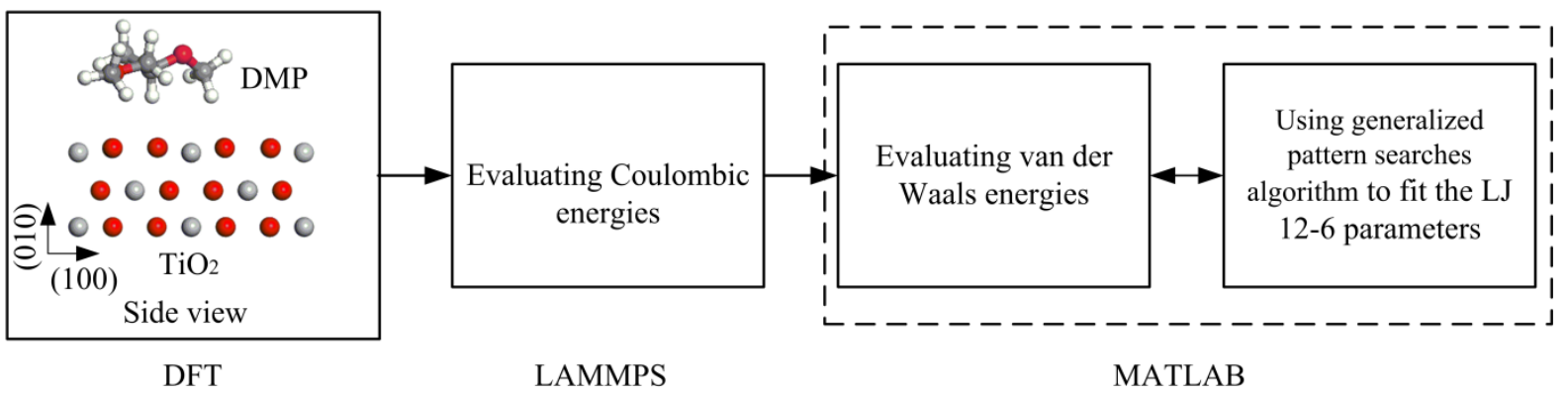

Fig. 2. LJ 12-6 parameters fitting procedure for deriving interaction parameters between PEO/PPO and $\mathrm{TiO}_{2}$ surface

\section{RESULTS}

\subsection{Fitted Interfacial Force Field}

As there are little experimental results available for the adsorption of 1,2-DME or 1,2-DMP on $\mathrm{Ti} / \mathrm{TiO}_{2}$ surface, the structural properties and adsorption energies obtained from DFT calculations have therefore been the reference data for training FF between copolymer and rutile surface. The best fitted function values of 2.91 and $7.28 \%$ were obtained respectively for 1,2-DME and 1,2-DMP after 1200 iterations. The vdW interaction energy for each adsorption configuration shown in Table 2 reveals that a good agreement between fitted FF and DFTD calculation has been found for 1,2-DME with the errors less than 5\%. The errors for 4 particular configuration of 1,2-DMP are around 10\%, and the rest of 6 configurations are less than $6.5 \%$ which is considered reasonable. The obtained fitted parameters for LJ 12-6 potential between 1,2-DME and $\mathrm{TiO}_{2}$ surface are given in Table 3 .

Table 2

Comparison of vdW interaction energy between fitted FF and DFTD

\begin{tabular}{lrccc}
\hline \multirow{2}{*}{ Molecule } & Fig. & \multicolumn{3}{c}{$-\mathrm{E}_{\text {ads }}(\mathrm{eV})$} \\
& DFTD & Fitted FF & Error (\%) \\
\hline $1,2-$ DME & $2 \mathrm{a}$ & 0.238 & 0.240 & 1.1
\end{tabular}




\begin{tabular}{ccccc} 
& 2b & 0.247 & 0.240 & -3.0 \\
2c & 0.238 & 0.240 & 1.0 \\
2d & 0.238 & 0.239 & 0.2 \\
2e & 0.240 & 0.241 & 0.1 \\
2f & 0.244 & 0.240 & -1.4 \\
2g & 0.261 & 0.250 & -4.1 \\
2h & 0.262 & 0.251 & -4.2 \\
2i & 0.173 & 0.182 & 4.9 \\
2j & 0.202 & 0.209 & 3.8 \\
\hline 3a & 0.402 & 0.399 & 0.8 \\
3b & 0.731 & 0.653 & -10.6 \\
3c & 0.470 & 0.448 & -4.7 \\
3d & 0.539 & 0.592 & 9.8 \\
3e & 0.419 & 0.408 & -2.5 \\
3f & 0.454 & 0.465 & 2.5 \\
3g & 0.478 & 0.530 & 10.8 \\
3h & 0.428 & 0.383 & -10.4 \\
3i & 0.845 & 0.882 & 4.4 \\
3j & 1.060 & 0.991 & -6.5 \\
\hline
\end{tabular}

${ }^{\mathrm{a}}$ DFT calculation and training configurations are presented in Supplementary Material (SM)

Table 3

Fitted LJ 12-6 parameters for interfacial interactions between 1,2-DME and 1,2- DMP with TiO2 surface.

\begin{tabular}{ccccc}
\multirow{2}{*}{ Pairwise } & \multicolumn{2}{c}{$1,2-\mathrm{DME}$} & \multicolumn{2}{c}{$1,2-\mathrm{DMP}$} \\
& $\varepsilon(\mathrm{eV})$ & $\sigma(\AA)$ & $\varepsilon(\mathrm{eV})$ & $\sigma(\AA)$ \\
\hline $\mathrm{Ti}-\mathrm{O}$ & 0.027300 & 2.362148 & 0.219391 & 1.722500 \\
$\mathrm{Ti}-\mathrm{C}$ & 0.036000 & 2.930273 & 0.036977 & 2.698828 \\
$\mathrm{Ti}-\mathrm{H}$ & 0.000100 & 4.499766 & 0.000100 & 3.517344 \\
$\mathrm{O}_{\mathrm{Ti}} \mathrm{O}$ & 0.008600 & 2.570703 & 0.086725 & 2.314844 \\
$\mathrm{O}_{\mathrm{Ti}} \mathrm{C}$ & 0.006500 & 3.162187 & 0.006500 & 3.049727 \\
$\mathrm{O}_{\mathrm{Ti}}-\mathrm{H}$ & 0.001900 & 2.36726 & 0.000923 & 2.617265 \\
\hline
\end{tabular}

\subsection{Low Weight Concentration}

The snap-shots of molecular structure in different simulation stages of Pluronic molecules are presented in Fig. 3 which shows that the triblock copolymers adsorb on rutile surface during three simulation stages. Many PPO blocks have been observed in regions close to the surface, whilst the PEO blocks are found at the outer region. The representative models of $\mathrm{L}$ and R Pluronics shown in Fig. 4 reveals a worm-like structure (anchor-buoy-anchor (A-B-A)) of 17R4 on the surface (Fig. 4a), whilst there is a brush-like structure (buoy-anchor-buoy (B-A-B)) for L62 (Fig. 4b). The worm-like structure was formed due to the adsorption of PPO blocks at both ends of copolymer onto the surface, while the hydrophilic PEO blocks with weaker adsorption strength were extended into water solution. The extended amount of PEO was not significant due to its small portion (20\%) in the molecule for 17R2 
and 25R2 (Fig. 3a, c), respectively. However, a larger portion has been observed for 17R4, which has a larger ratio of PEO (40\%) block in the molecule. These observations indicate that the worm-like structure depends on the percentage of PEO in an R-Pluronic molecule.

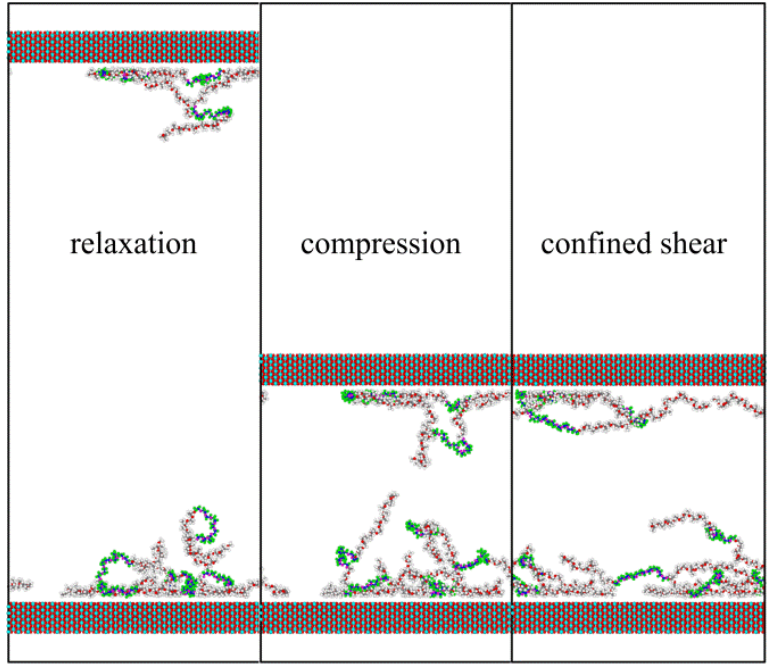

(a) $17 \mathrm{R} 2-2 \%$

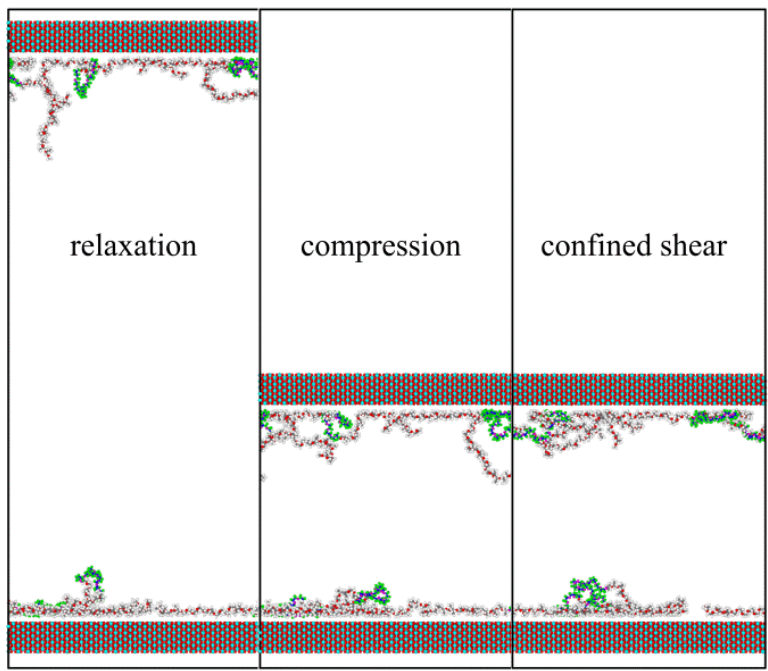

(c) $25 \mathrm{R} 2-2 \%$

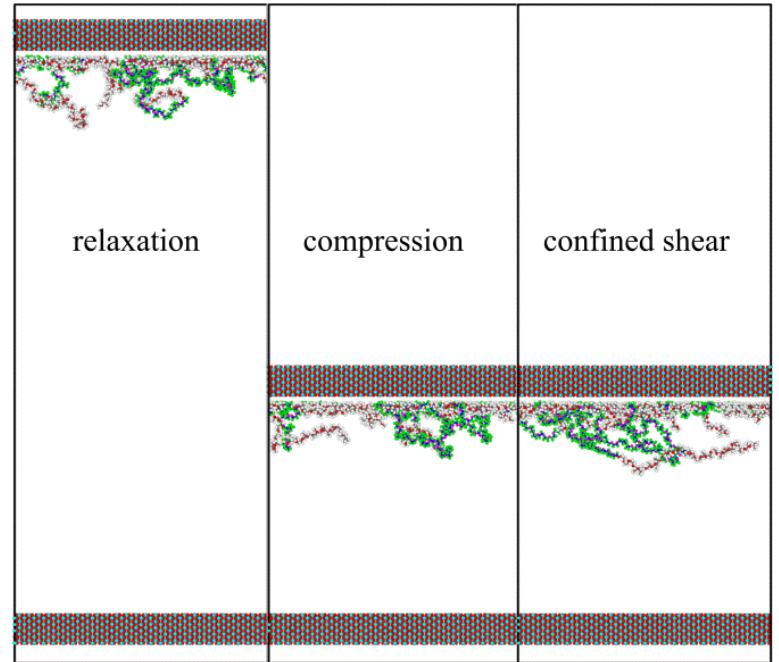

(b) $17 \mathrm{R} 4-2 \%$

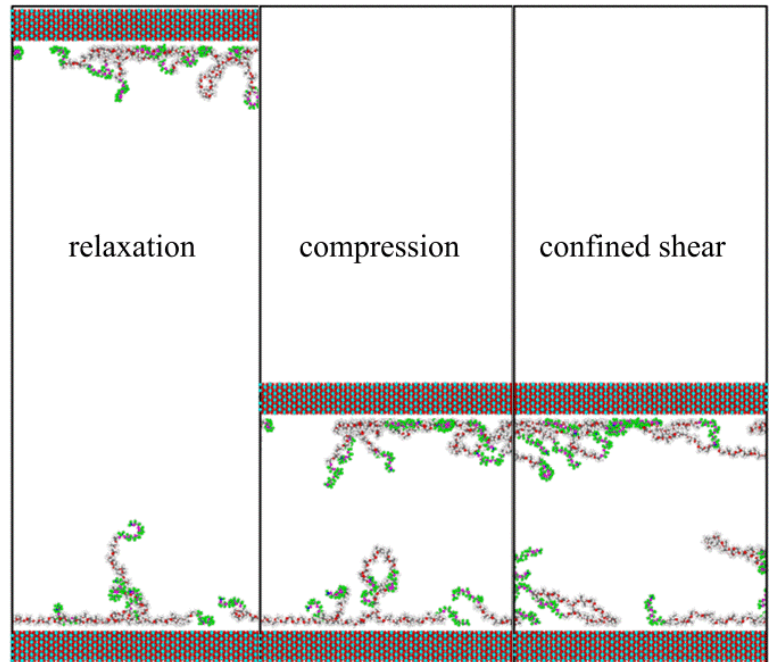

(d) $\mathrm{L} 62-2 \%$

Fig. 3 Molecular structures of: (a) 17R2; (b) 17R4; (c) 25R2, and (d) L62 triblock copolymers (2\%) confined between $\mathrm{TiO}_{2}$ surfaces at $300 \mathrm{~K}$ after relaxation (6 ns), compression (10 ns), and confinedshear (14 ns).

The mass density profiles across the film thickness during relaxation are plotted in Fig. 5 to analyze further the molecular structure of the considered copolymers. This density profile was calculated by evaluating the atomic mass density in a lubricant slab $(0.311 \AA)$ in $\mathrm{z}$ direction. The results show that a larger density of copolymer has been found at solid-fluid interface. A larger amount of atomic density of PPO blocks is located at the lubricant- $\mathrm{TiO}_{2}$ interface. It is also noted that this density profile is not symmetrical. This phenomenon has also been observed from previous studies [27, 34], and it could be explained by the non-homogeneous solution at atomic scale [34]. 


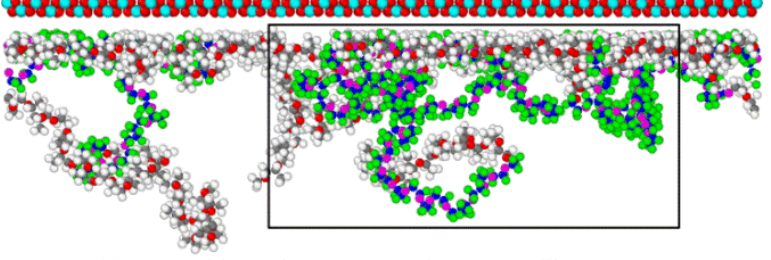

(a) 17R4 with Anchor-Buoy-Anchor (worm-like structure)

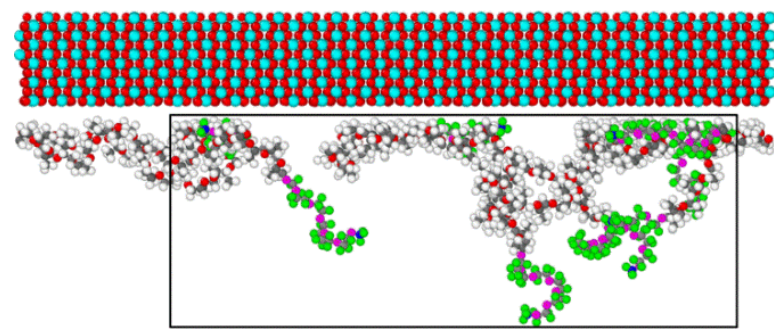

(b) L62 with Buoy-Anchor-Buoy (brush-like structure)

Fig. 4 Molecular structures of: (a) thin 17R4 film adsorbed on bottom $\mathrm{TiO}_{2}$ surface; and (b) thin $\mathrm{L} 62$ film adsorbed onto top $\mathrm{TiO}_{2}$ surface after relaxation at $300 \mathrm{~K}$ at $6.0 \mathrm{~ns}$.
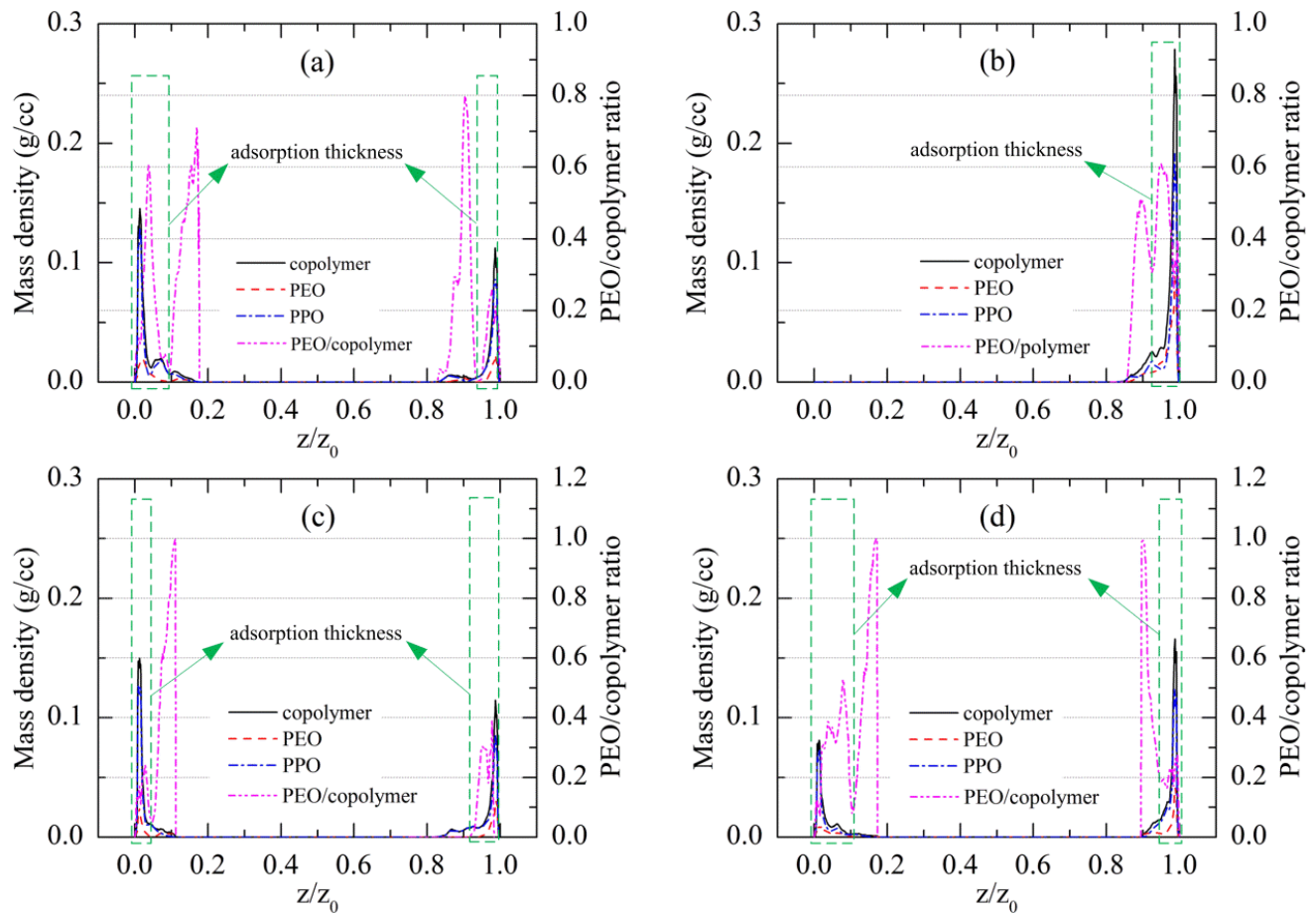

Fig. 5 Density profile across the film thickness of: (a) 17R2; (b) 17R4; (c) 25R2, and (d) L62 triblock copolymers $(2 \%)$ during relaxation stage. 

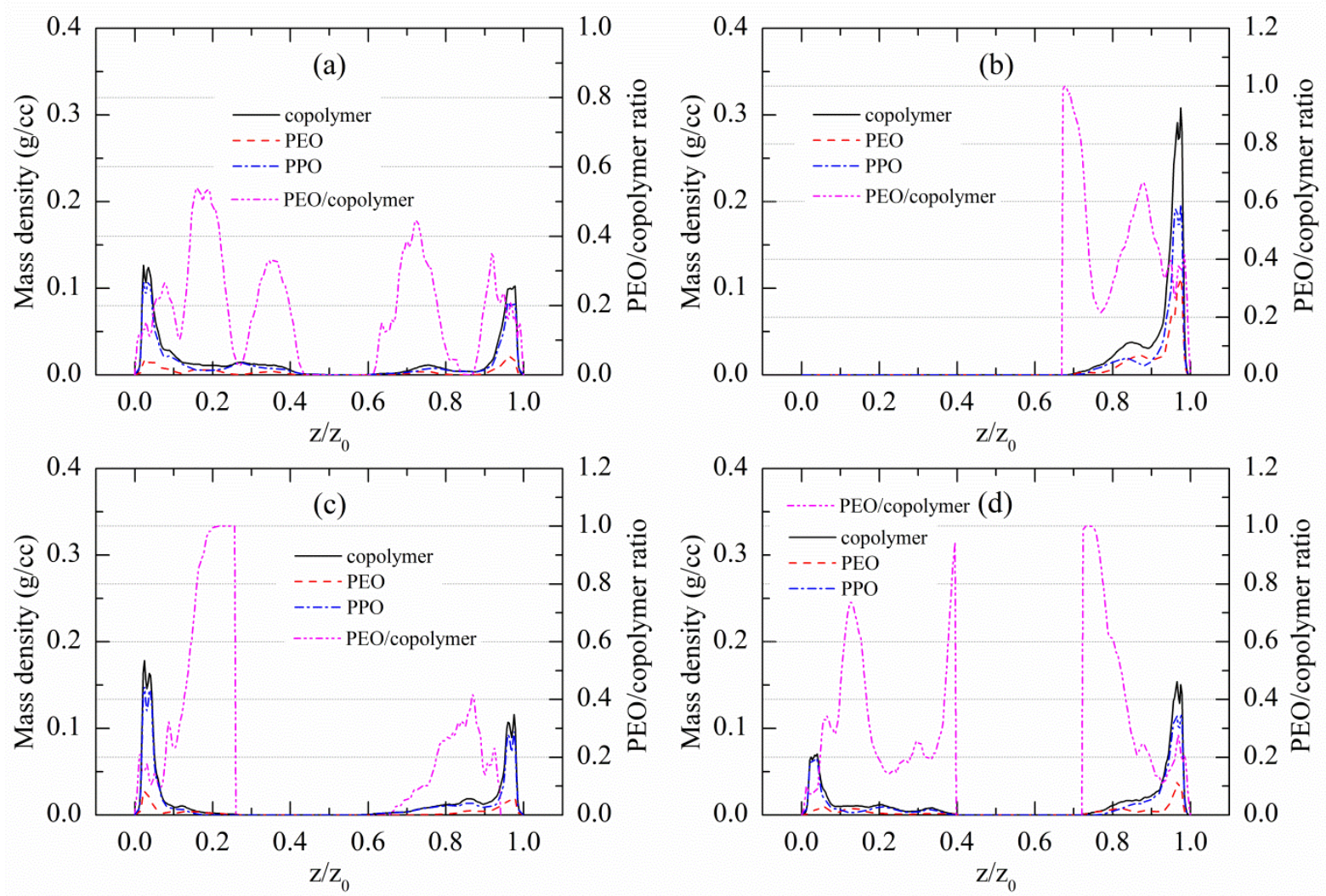

Fig. 6 Density profile across the film thickness of: (a) 17R2; (b) 17R4; (c) 25R2, and (d) L62 triblock copolymers $(2 \%)$ during compression stage.

The applied pressure yields a small enhancement of adsorbed layers. In fact, Fig. 6 indicates that the peaks of density profile at solid-fluid regions during compression are higher than those obtained from relaxation in Fig. 5. This finding reveals an agreement with another MD investigation on the influence of applied pressure for hydrocarbon lubricant [37]. The density profile in Fig. 7 for confined shear process shows that there is a small reduction in the peak of density profile at solid-fluid interface for 17R2, while this density profile is retained for other cases. Additionally, the snap-shot of copolymer adsorbed on the rutile surface presented in in Fig. 8 also shows that the copolymers do not align in shearing direction. This behavior is contrasting with that found for homogeneous hydrocarbon $[38,51]$. These observations reveal a fact that the shear does not affect significantly the molecular structure of the adsorbed triblock copolymer at low copolymer concentration. 

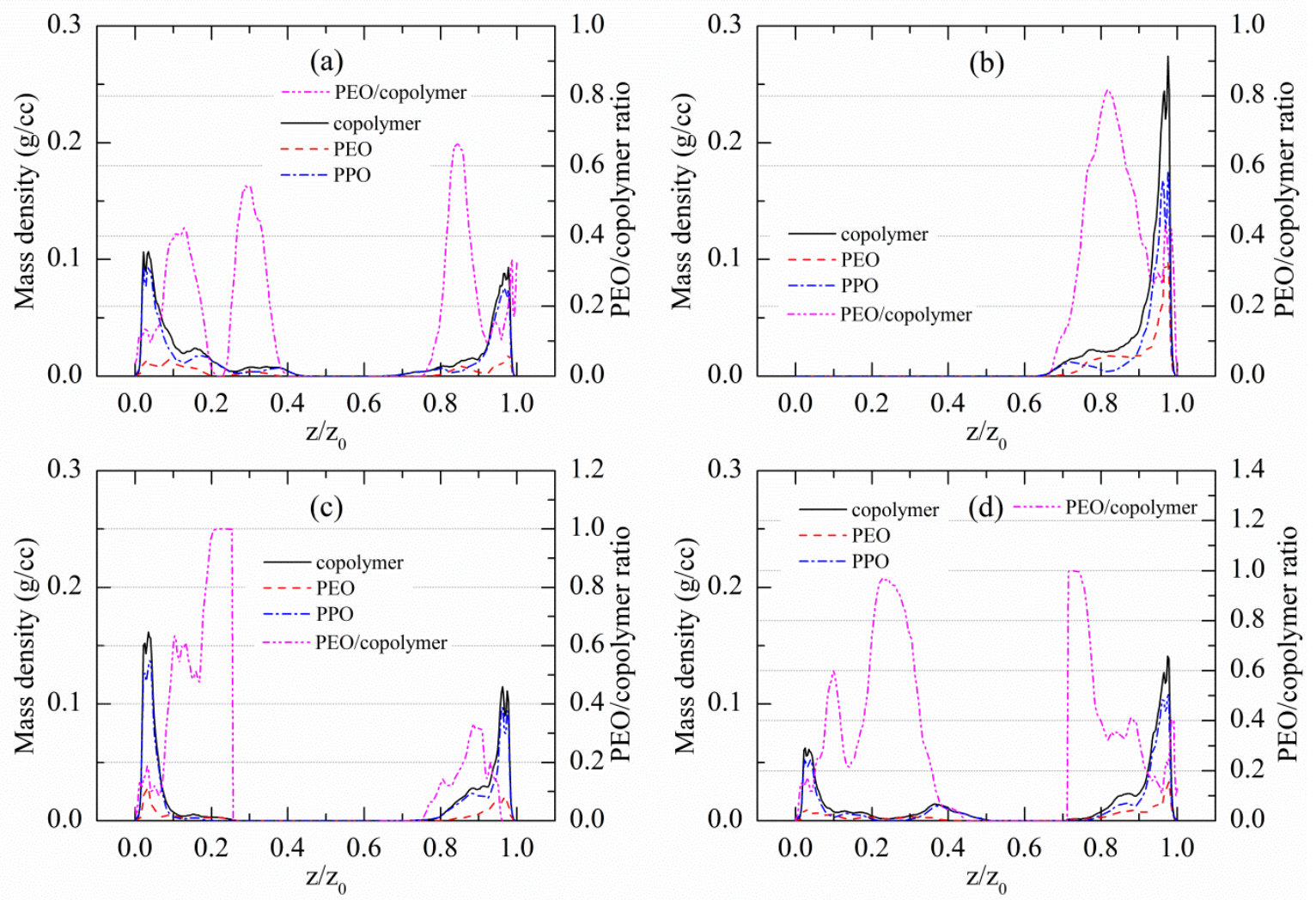

Fig. 7 Density profile across the film thickness of: (a) 17R2; (b) 17R4; (c) 25R2, and (d) L62 triblock copolymers $(2 \%)$ during confined shear stage.

The molecular structure in Fig. 3-4 and the statistical results in Fig. 5-7 show that there is still a small amount of PPO present at the outer layer and vice versa, a significant amount of PEO has been found at the solid-fluid interfaces. To quantify the dominant regime of these blocks, the ratio of atomic mass density of PEO to copolymer has been defined and plotted in these figures. The zero value means there is no PEO, while the ratio of 1 reveals that there is only PEO in that region. The standard ratio of 0.2 (20\% of PEO) is for 17R2, 25R2, and L62 molecules, and 0.4 for 17R4. However, these figures show that the significant higher ratio has been found in regions beyond the solid-fluid interfaces. These statistical results again confirm that the PEO block is the dominant part present at the outer regime of the adsorbed thin copolymer film at solid-fluid interfaces.

The adsorption thickness was defined as the distance from the surface to the transition position between the first peak and the second one of $\mathrm{PEO} /$ polymer density-ratio profile during relaxation. This transition position was the termination of the first buoy PEO layer. Fig. 5 describes clearly how this adsorption thickness was measured for each simulated system. This thickness measurement will be compared with previous experimental measurements in which the influence of applied pressure or sliding velocity was not considered $[15,20]$. Moreover, the adsorption thickness was averaged from both top and bottom 
surfaces, if the copolymers adsorbed on both surfaces. The thicknesses for 17R2, 17R4, 25R2, and L62 were $19.13,20.22,16.33$, and $18.71 \AA$ A respectively.
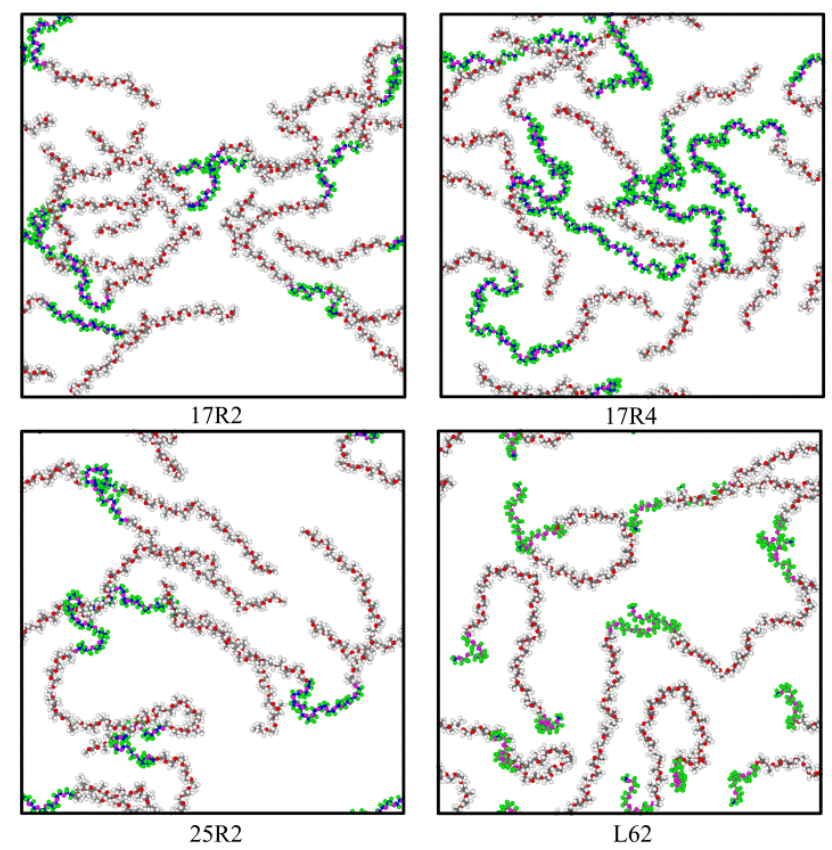

Fig. 8 Top-views of molecular structure of 17R2, 17R4, 25R2, and L62 triblock copolymers on $\mathrm{TiO}_{2}$ surface after confined shear process

The time evolution of adsorption energies (- $\left.\mathrm{E}_{\mathrm{ad}}\right)$ for copolymers and their constituent blocks are presented in Fig. 9. These energies increase gradually during the relaxation stage and become stable after $3 \mathrm{~ns}$, which prove that the total simulation time of $14 \mathrm{~ns}$ should be enough to obtain reasonable statistical results. The average adsorption energies (Fig. 9d) and their corresponding energies per molecule (Fig. 10a) increase with the chain length for R Pluronics in following order 17R2 < 17R4 < 25R2, while L62 show a remarkable lower values despite it has similar molecular weight with 17R4 and similar percentage of PEO with 17R2. The individual contributions of adsorption energy of constituent parts of PEO and PPO increase with their number in the molecule in an order of 17R4 < 17R2 < 25R2 for PPO and 17R2 < 25R2 < 17R4 for PEO (Fig. 9d).

The chart in Fig. 10a reveals the contribution of PEO and PPO blocks in total adsorption energy of a copolymer molecule. The adsorption energy per molecule of PEO/PPO block has been evaluated by dividing its relevant total adsorption energy by the number of copolymer molecules. This chart shows an increase of adsorption energy per molecule of PPO block with molecular chain length for $\mathrm{R}$ copolymers. However, this propensity is not observed for PEO, and the highest adsorption energy per PEO block is found for 17R4 which has the highest PEO/PPO ratio. For L62, these energies (Fig. 10a, b) are similar to the values evaluated for 17R2, due to their similarity in molecular chain length and $\mathrm{PEO} / \mathrm{PPO}$ ratio in the molecule. 
The results from Fig. 9d and 10a show that the total adsorption energy and the adsorption energy per copolymer molecule increase with the molecular chain length. This interesting observation has raised a question of whether or not it is a linear relationship. The adsorption energy per PEO/PPO block in each copolymer has been evaluated to investigate this phenomenon. This value is calculated by dividing the adsorption energy per molecule of PEO/PPO by its number of blocks in the copolymer. Fig. 10b shows that the energy is not similar or all copolymer and it increases with the chain length in R Pluronics. This trend is also observed for each individual constituent part of PEO and PPO blocks. These findings indicate the fact that the increase of the total adsorption energy and the adsorption energy per molecule with chain is not linear.
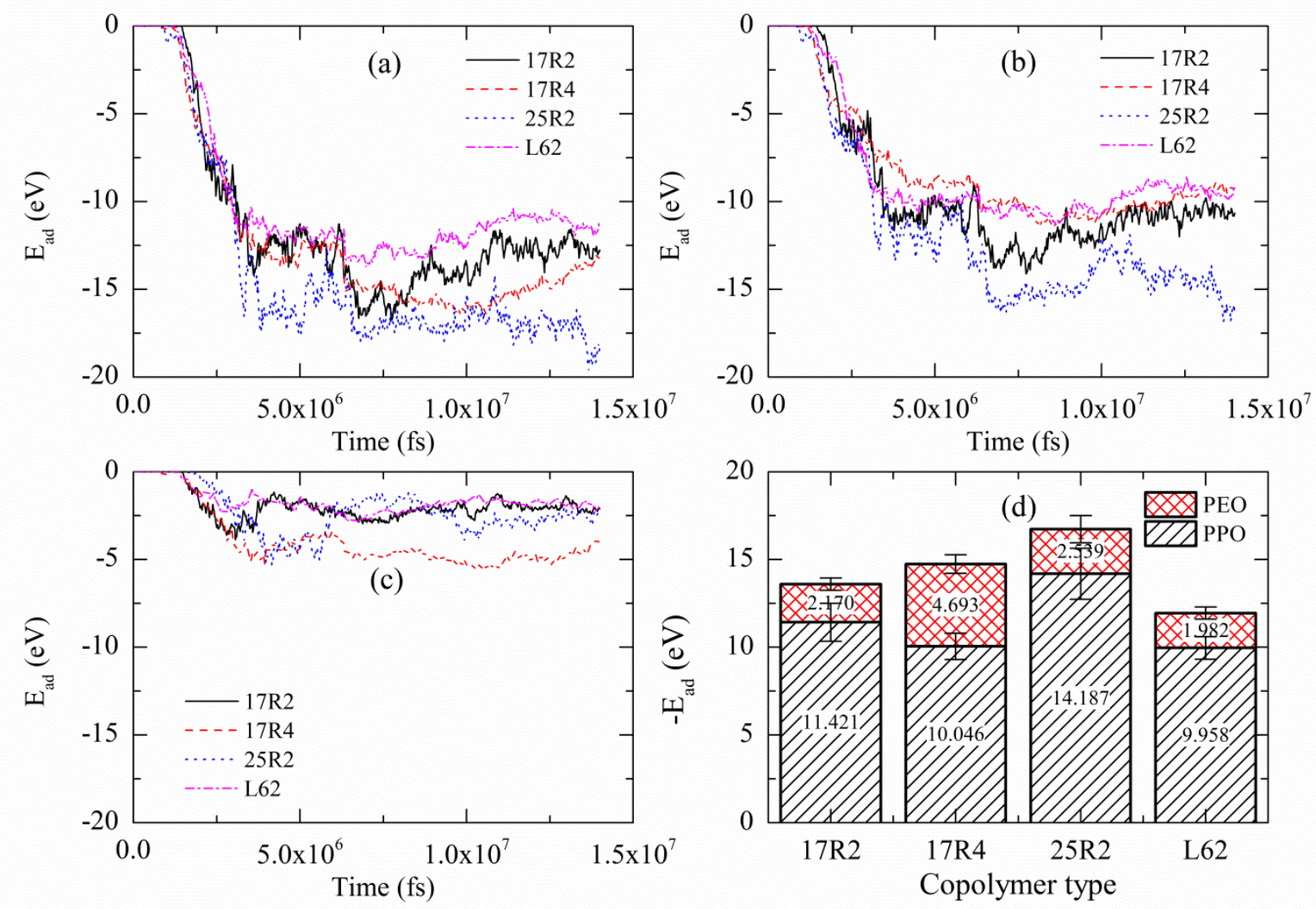

Fig. 9 Time evolution of adsorption energies of: (a) copolymer; (b) PPO; (c) PEO; and (d) average adsorption energies for each type of copolymer (2\%) in aqueous solution. 

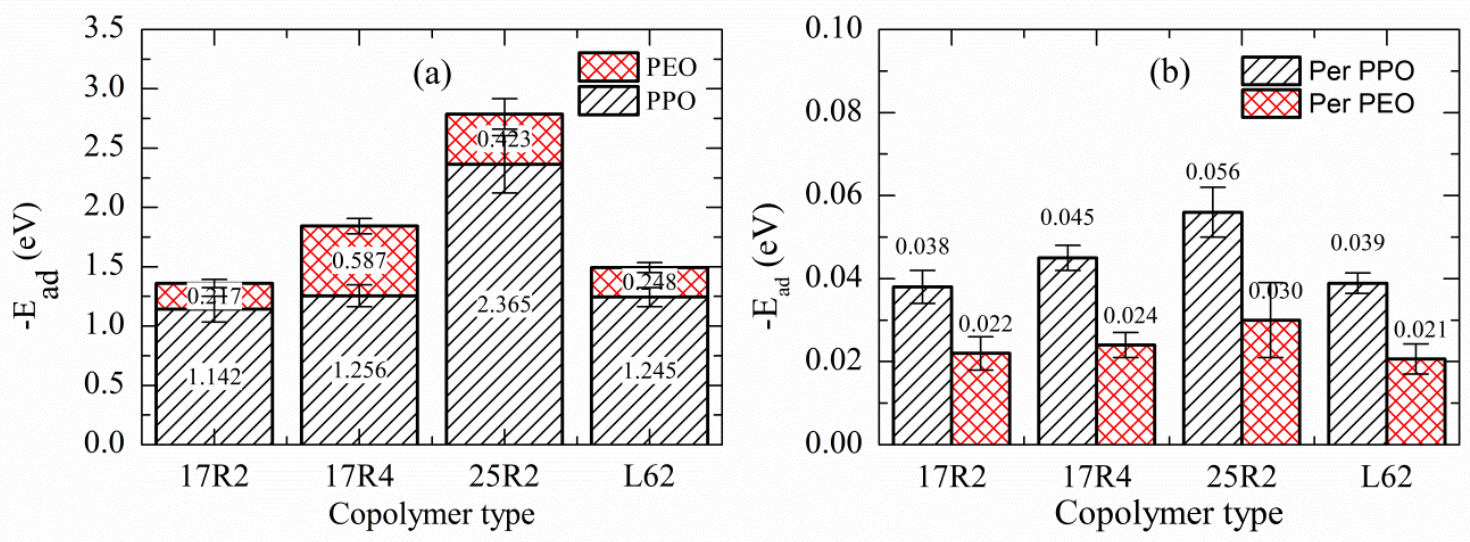

Fig. 10 (a) Individual contributions of PEO and PPO in total adsorption energy per molecule; and (b) specific adsorption energy per PEO/PPO group for different types of copolymer in aqueous solution.

The rheological properties were also investigated and presented in Fig. 11. The time evolution of shear stresses presented in Fig. 11a show a large deviation of shear stress compared with their average values. This shear stress $\left(\tau_{x z}\right)$ was measured by the shearing force exerted by the interactions of lubricant molecules to surface atoms on the surface area. The averaged shear stresses of $0.5-2.671 \mathrm{MPa}$ for these copolymers are insignificant. These values are small compared with their deviations of 15.68-16.79 MPa. The magnitudes of these deviations were commonly observed in MD simulation [37, 38, 52], and they were influenced by many factors such as the surface corrugation [38], as well as the temperature and applied pressure [37]. The density profiles across the film thickness as presented in Fig. 11c show that there is no slip at the solid-fluid interfaces. Additionally, the lubricants behave more liquid-like with a linear gradient profile of lubricant velocity. This characterization is also contrasting with that observed for hydrocarbon lubricant [37, 38].

Finally, the shear viscosities that characterized for rheological properties of lubricant are revealed in Fig. 11d. The viscosity $(\eta)$ in the shear flow is defined by

$$
\eta=\frac{\tau_{x z}}{\dot{\gamma}}
$$

where $\dot{\gamma}$ denotes the shear rate. The average viscosities of $0.25-1.336 \mathrm{mPa} . \mathrm{s}$ have been evaluated for these lubricants. The current calculated viscosities of the considered lubricants with $2 \%$ triblock copolymer are close to the experimental measured value of $\sim 1.2 \mathrm{mPa} . \mathrm{s}$ for $0.06 \mathrm{~g} / \mathrm{ml}$ copolymer concentration and 300K [53]. However, large deviations of 7.844-8.394 mPa.s have been found for these calculated viscosities. This is the consequence of the large deviations in shear stress that are shown in Fig. 11b. An interesting observation is that L62 copolymer has the lowest shear stress and viscosity 
than other R Pluronics, which shows an agreement with a previous experiment that revealed the lowest coefficient of friction of L62 (0.24) compared with 17R4 (0.39) and $25 \mathrm{R} 2(0.29)$ at $25^{\circ} \mathrm{C}$ [1].
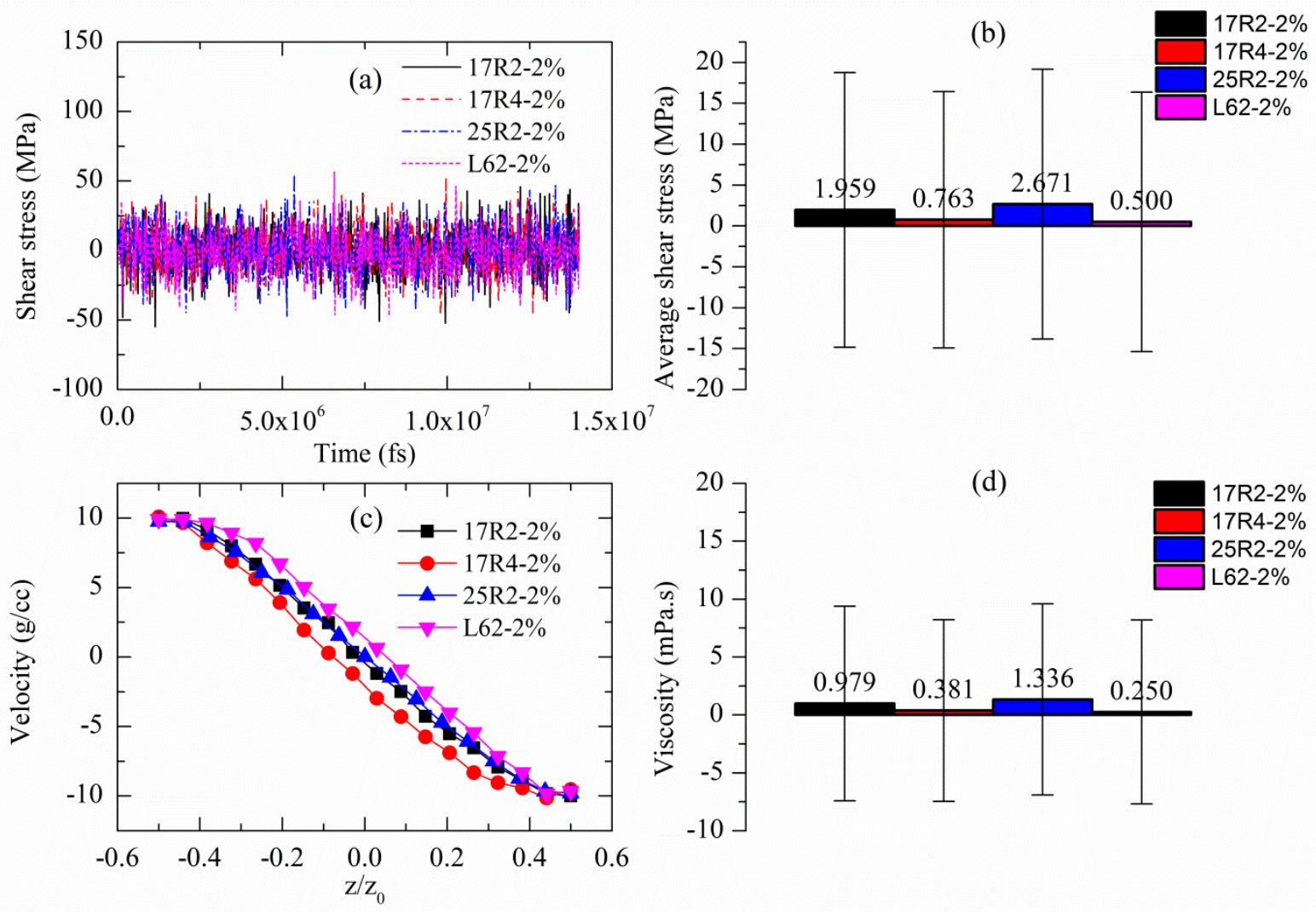

(d)

$17 R 2-2 \%$ $17 \mathrm{R} 4-2 \%$ $25 \mathrm{R} 2-2 \%$ L62-2\%

Fig. 11 (a) Time evolution of shear stresses; (b) average shear stresses; (c) velocity profiles across the film thickness; and (d) shear viscosities for different triblock copolymer (2\%) lubricants

\subsection{High Weight Concentration}

The snap-shots of molecular structure at the end of each simulation stage for different copolymers at high concentration are presented at Fig. 12. At high temperature and vacuum condition, the copolymers gradually adsorb onto rutile surface without forming micelle structure. A larger amount of copolymer presents at solid-liquid interfaces than at inner region of the lubricant. Under the shear effect, the copolymers in the middle region of the lubricant are stretched in the shearing direction. It is noted that there is no separate phase of PEO and PPO blocks at low concentration. These blocks are found everywhere in the lubricant. Moreover, the A-B-A and B-A-B are not explicitly observed on the surface. These facts reveal that the high concentration of copolymer yields a change in the adsorption behavior of copolymers.

The density profile during relaxation has been plotted in Fig. 13 to calculate the adsorption thickness at a high copolymer concentration. The measurement was similar to that at low concentration. The 
evaluated thicknesses for 17R2, 17R4, 25R2, and L62 were 26.65, 20.68, 21.56, and $20.53 \AA$ respectively. A comparison of density profile of $17 \mathrm{R} 4$ during compression and confined shear has been carried out to investigate the influence of the shear effect on the molecular structure of the copolymer at high concentration. Fig. 14b shows that the peaks of density profile at solid-fluid interfaces during shearing have been reduced compared with that obtained in the compression stage (Fig. 14a). The strong adhesion of copolymer with the surface has yielded a movement of the copolymers at solid-fluid interfaces that reduces the adsorption of these copolymers and consequently, the density of copolymer. In fact, the snap-shot of the molecular structure of 17R4 in the shearing stage in Fig. 12b shows the molecules are stretched due to the shear. Additionally, Fig. 14b also shows the larger copolymer density in the middle region of lubricant during the shearing process than that during the compression stage. This reveals that the copolymers have migrated from solid-fluid interfaces into the middle region of the lubricant. Similar observations are also found for other copolymers in Fig. 15, 16. Furthermore, a layering structure of copolymer has been found on the surface with a significantly larger density of PPO than PEO. The averaged peak of density of $\sim 0.4 \mathrm{~g} / \mathrm{cc}$ has been found at the solid-fluid interface for these copolymers. This value has been reduced by an amount of $0.05 \mathrm{~g} / \mathrm{cc}$ during the shearing process. 


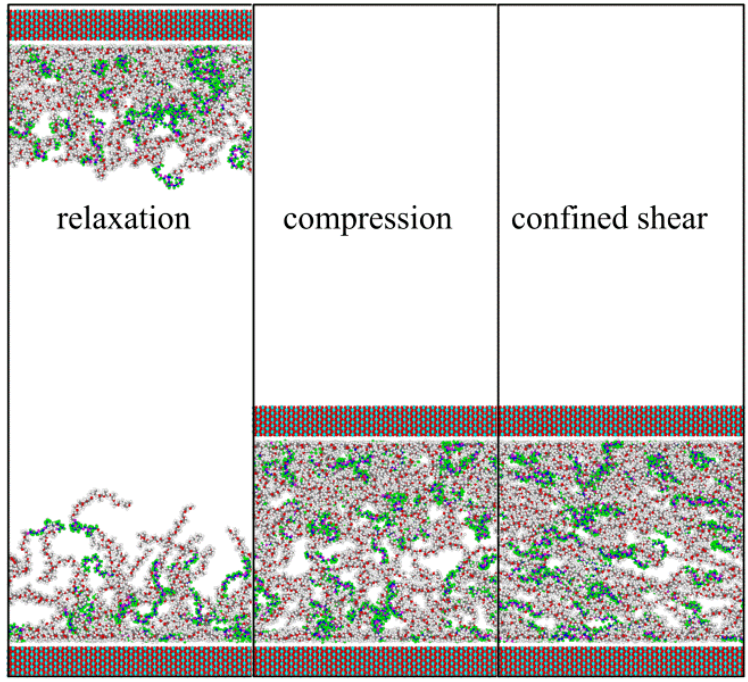

(a) $17 \mathrm{R} 2-16 \%$

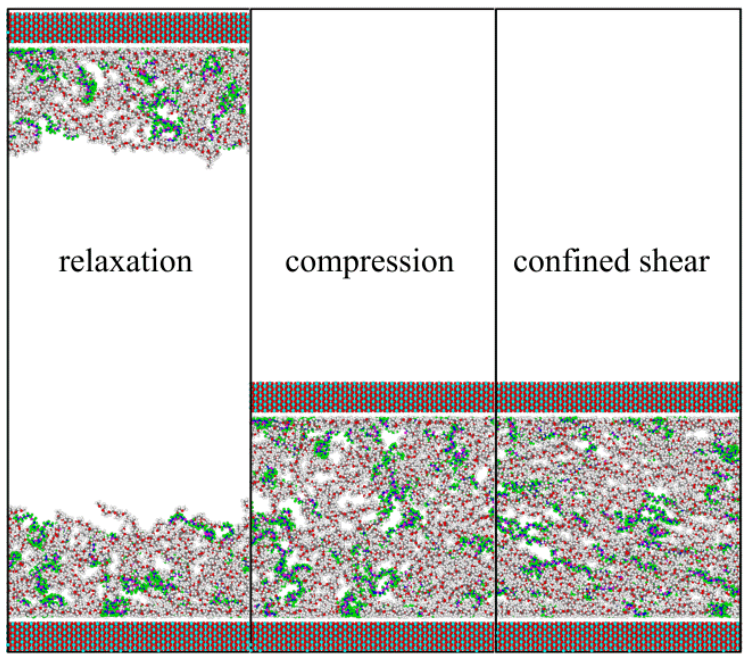

(c) $25 \mathrm{R} 2-16 \%$

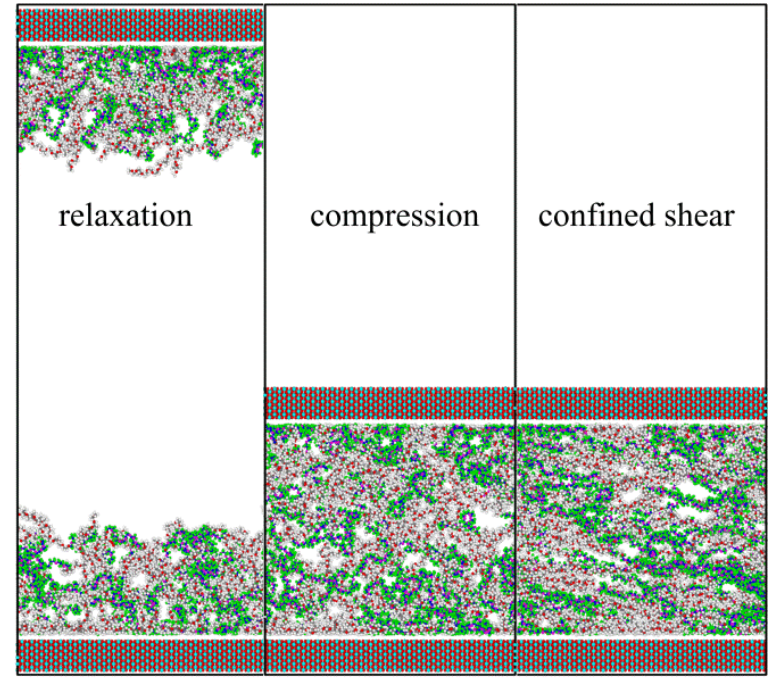

(b) $17 \mathrm{R} 4-16 \%$

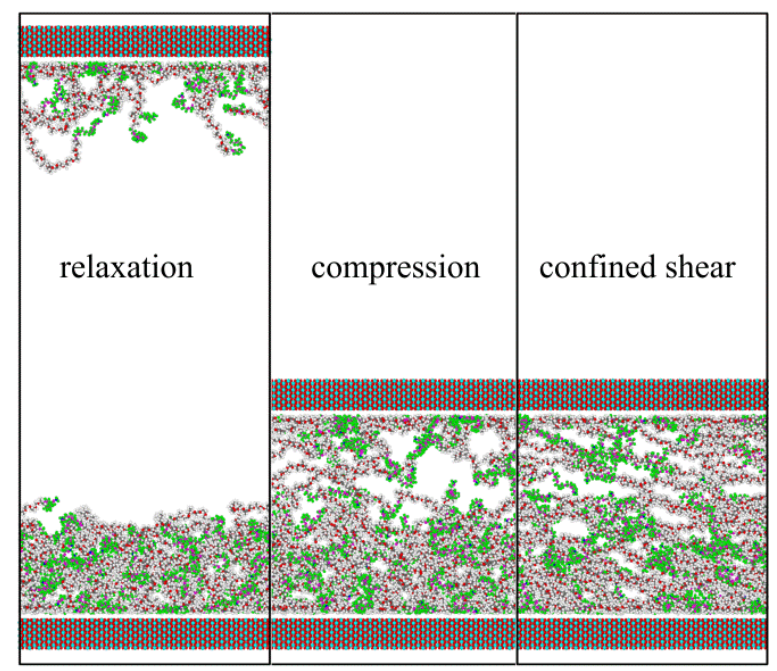

(d) $\mathrm{L} 62-16 \%$

Fig. 12 Molecular structures of: (a) 17R2; (b) 17R4; (c) 25R2, and (d) L62 triblock copolymers (16\%) confined between $\mathrm{TiO}_{2}$ surfaces at $300 \mathrm{~K}$ after relaxation (6 ns), compression (10 ns), and confinedshear (14 ns). 

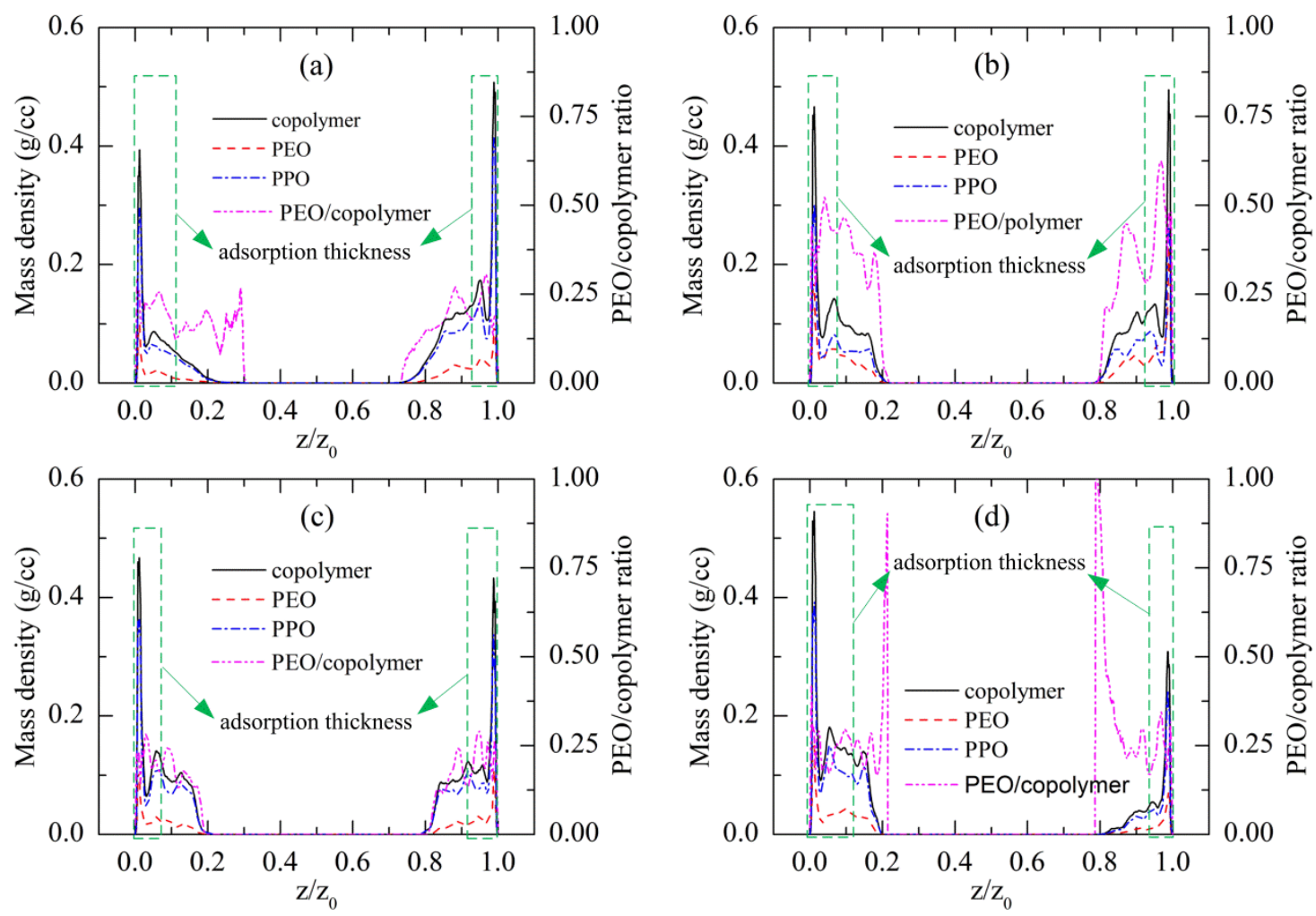

Fig. 13 Density profile across the film thickness of: (a) 17R2; (b) 17R4; (c) 25R2, and (d) L62 triblock copolymers $(16 \%)$ during relaxation stage.
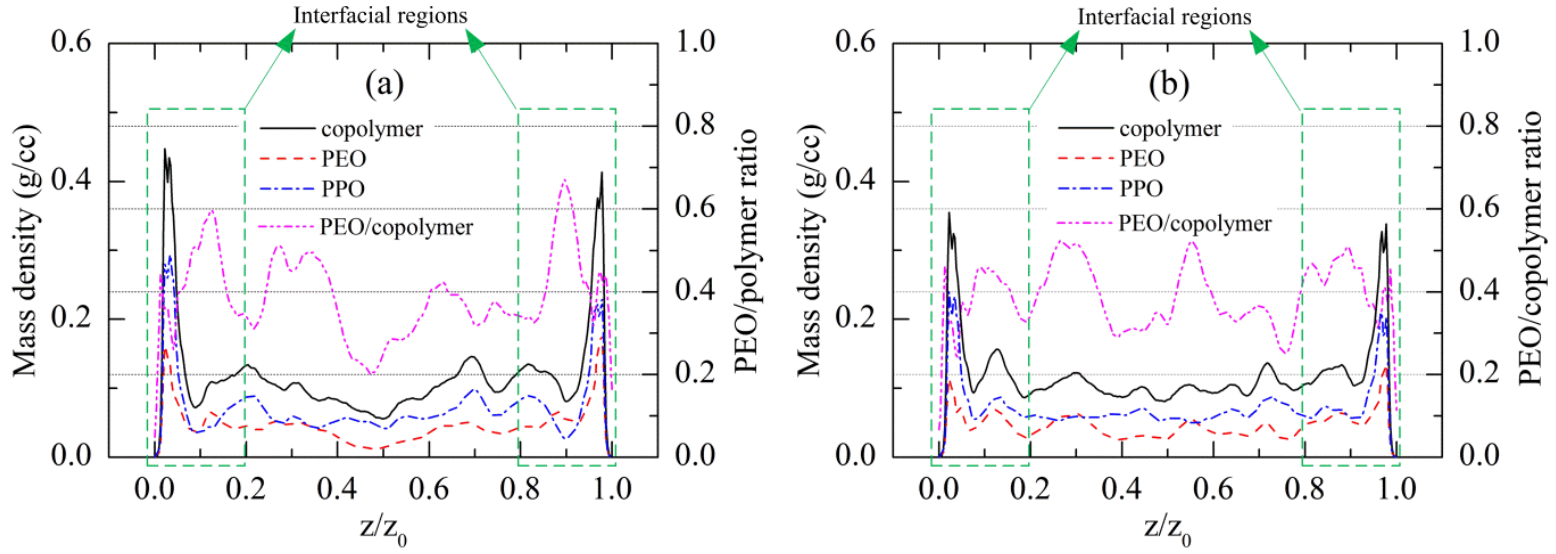

Fig. 14 Density profile across the film thickness of 17R4 (16\%) during: (a) compression stage, and (b) confined-shear stage.

The sliding of the surfaces has reduced the adsorbed mount of copolymer on the surface in a propensity that the higher is the ratio of PEO block, the larger is the amount of reduction. In fact, Fig. 15 provides the average peaks of density profile during the compression of $0.465,0.430,0.426$, and $0.413 \mathrm{~g} / \mathrm{cc}$ for 17R2, 17R4, 25R2, and L62. These values have been reduced during the shearing process and the results of $0.415,0.347,0.389$, and $0.387 \mathrm{~g} / \mathrm{cc}$ have been obtained in Fig. 16. The relevant reduction amounts 
of $0.050,0.084,0.037$, and $0.025 \mathrm{~g} / \mathrm{cc}$ were estimated for these copolymers. Clearly, 17R4 results in the largest reduction, whilst L62 yields an insignificant reduction. Furthermore, 25R2 with a longer molecular chain gives a smaller reduction than $17 \mathrm{R} 2$ despite the fact that they have a similar ratio of $\mathrm{PEO} /$ polymer.
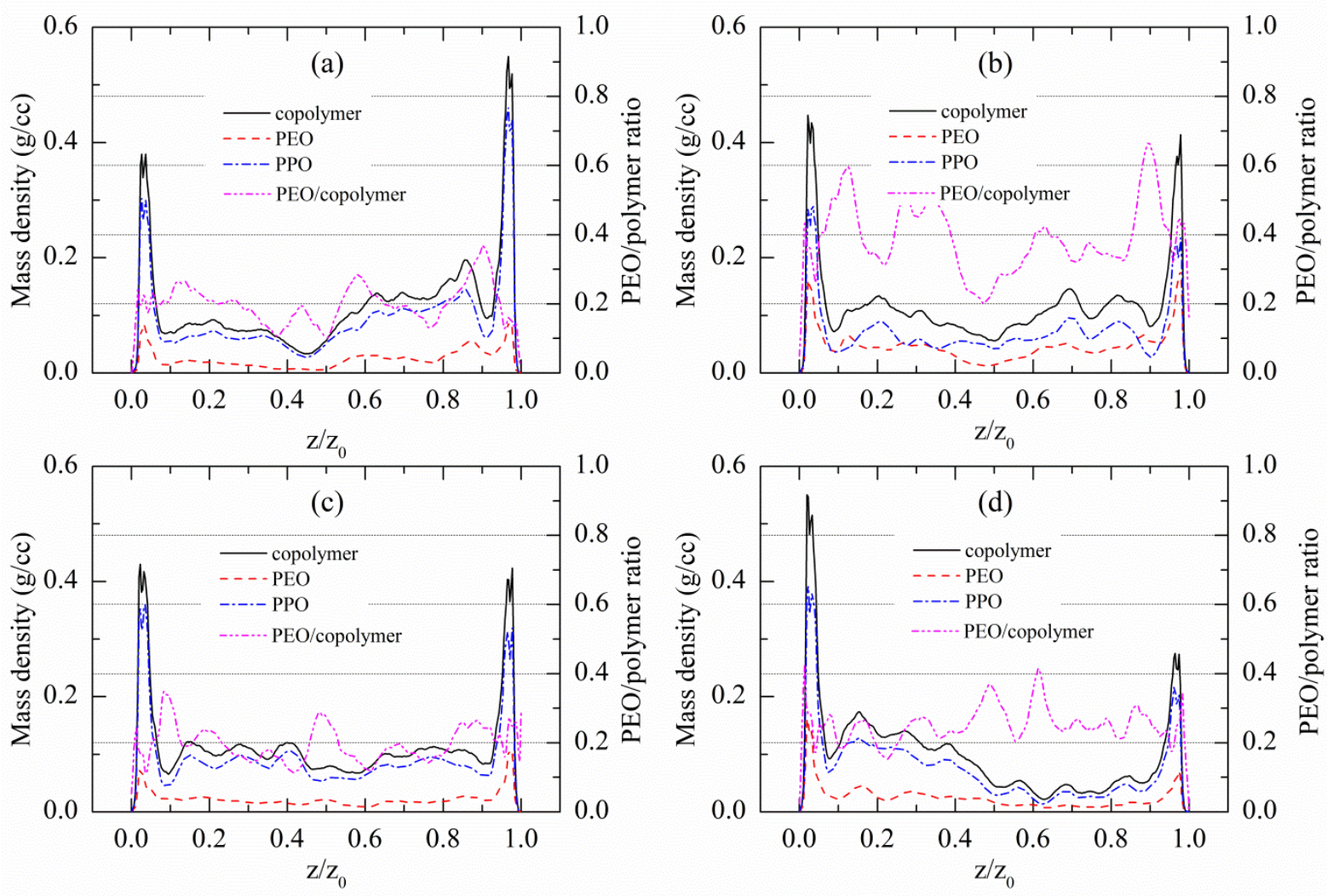

Fig. 15 Density profile across the film thickness of: (a) 17R2; (b) 17R4; (c) 25R2, and (d) L62 triblock copolymers $(16 \%)$ during compression stage.

The density ratio of PEO/copolymer on the density of copolymer at the interfacial region as shown in Fig. 12-15 shows that this ratio is close to the first layer of triblock copolymer at solid-fluid interfaces, and is still larger than its standard value in the copolymer molecule. This observation is similar to that at low concentration. However, the difference of PEO/polymer ratio between the first-layer region and its neighbor is not significant compared with that observed at low concentration. This observation could be explained by the fact that the triblock copolymers tend to adsorb onto the surface due to their strong adhesion strength. When the surface is fully covered, the copolymers would form the sub-layers with a lower atomic mass density and be allocated at regions adjacent to the first layer. The presence of PPO blocks at these adjacent layers has consequently reduced the ratio of PEO/copolymer in these regions. In contrast, these sub-layers were not formed at low concentration due to the limited number of molecules that was not enough to cover completely the surface. 

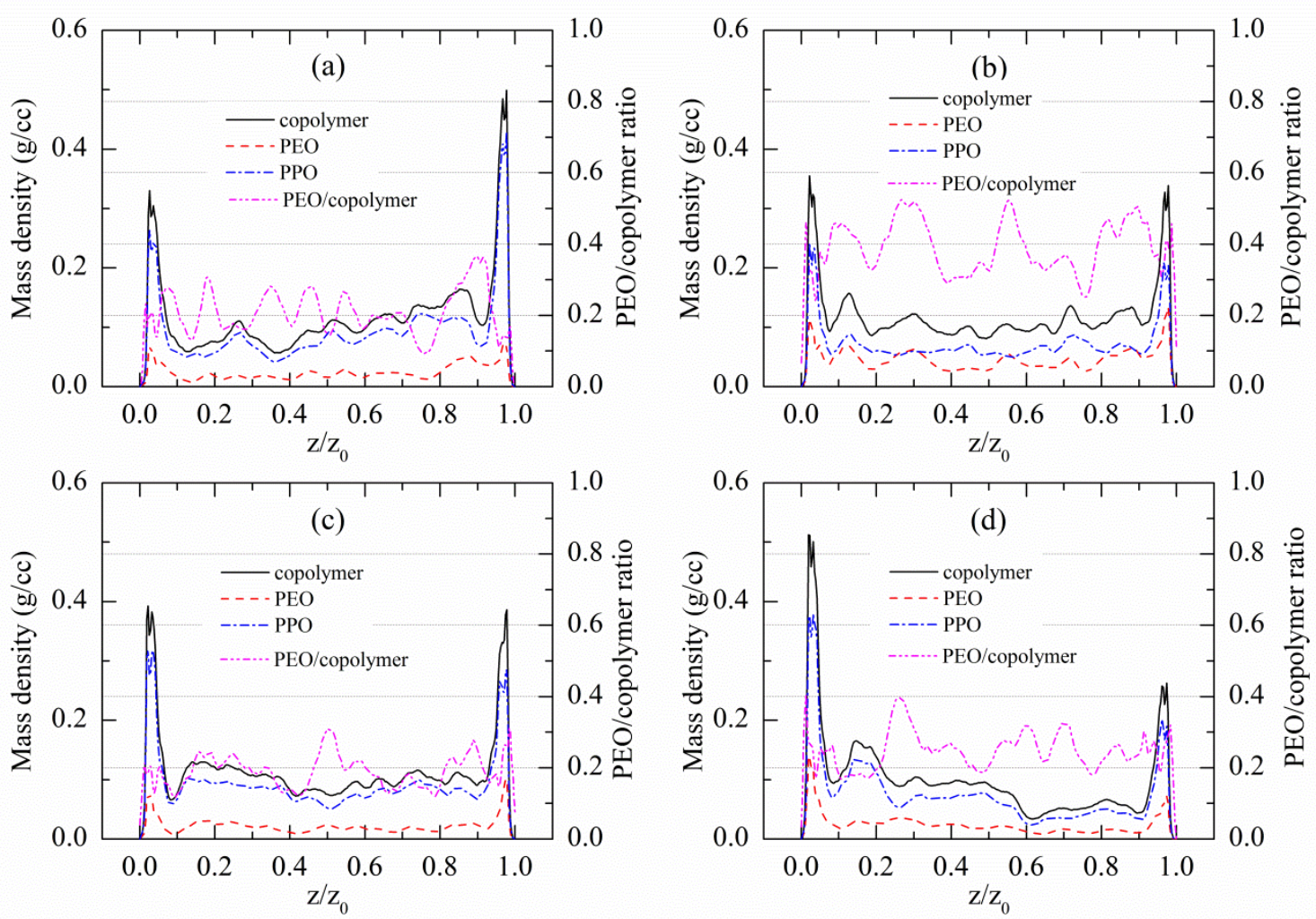

Fig. 16 Density profile across the film thickness of: (a) 17R2; (b) 17R4; (c) 25R2, and (d) L62 triblock copolymers (2\%) during confined shear stage.

The time evolution of adsorption energy of different copolymer (Fig. 27a) and their constituent blocks (Fig. $17 \mathrm{~b}, \mathrm{c}$ ) are also considered. The curves show that there is a decrease of adsorption energy with simulation time during the shear process from 10 to $14 \mathrm{~ns}$. The reduction of adsorption of copolymers on the surface discussed in previous paragraphs is the main reason for this phenomenon. It is interesting that Fig. 17d shows an insignificant difference of average adsorption energies between different copolymers at high concentration. This observation is contrasting with that observed at low concentration. The largest total adsorption energy of $23.25 \mathrm{eV}$ has been found for 17R2, while the lowest value of $21.22 \mathrm{eV}$ has been measured for 17R4. Only a small energy difference of $8.7 \%$ has been determined between them. However, this figure shows an agreement with the observation at low concentration, that the energy contribution of individual blocks is almost similar to its ratio in the molecule. 

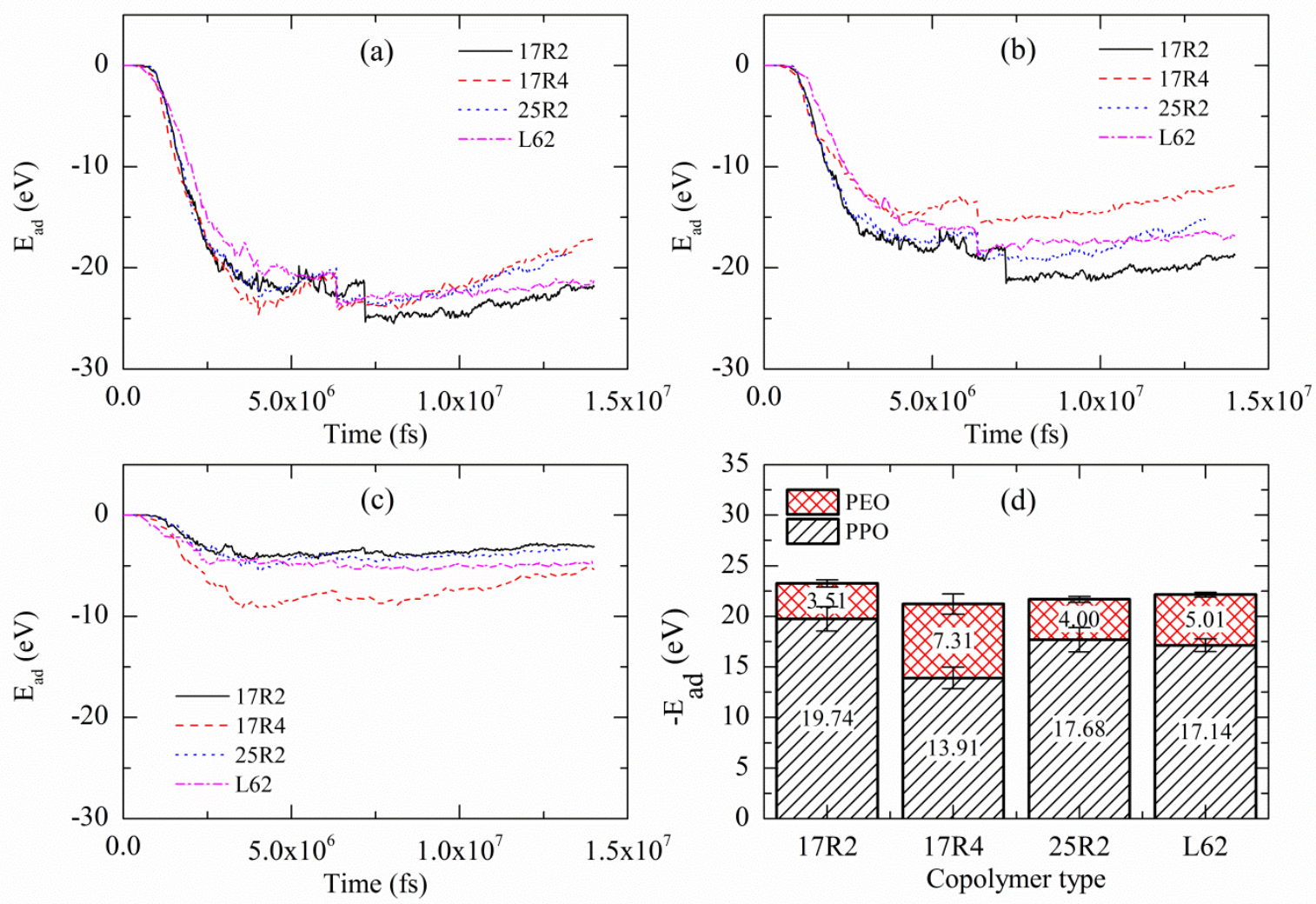

Fig. 17 Time evolution of adsorption energies of: (a) copolymer; (b) PPO; (c) PEO; and (d) average adsorption energies for each type of copolymer (16\%) in aqueous solution.

The rheological properties of aqueous lubricants are presented in Fig. 18. The time evolution of shear stress of these lubricants is presented in Fig. 28a. Their averaged values in Fig. 28b and the viscosities in Fig. 18d reveal an order of L62 < 17R2 <25R2 < 17R4. At high concentration, the velocity profile in Fig. 18c shows a nonlinear relationship between the velocity and film thickness and it is contrasting with that observed at low concentration. The effective shear rate presented in Fig. 18d is fitted based on the velocity profile. The fitted result shows a higher value compared with the apparent one that was evaluated based on the sliding velocity of both surfaces. This phenomenon is commonly observed for organic lubricants such as hydrocarbon [37, 38]. In fact, the fitted lines in Fig. 18c show that the effective shear rates are 1.38-1.52 times larger than the apparent ones. Consequently, the effective shear viscosities are less than the apparent ones by the same factors. Although there is a reduction of effective shear viscosity compared with the apparent one, the order of viscosity of L62 < 17R2 < 25R2 < 17R4 is still preserved. 

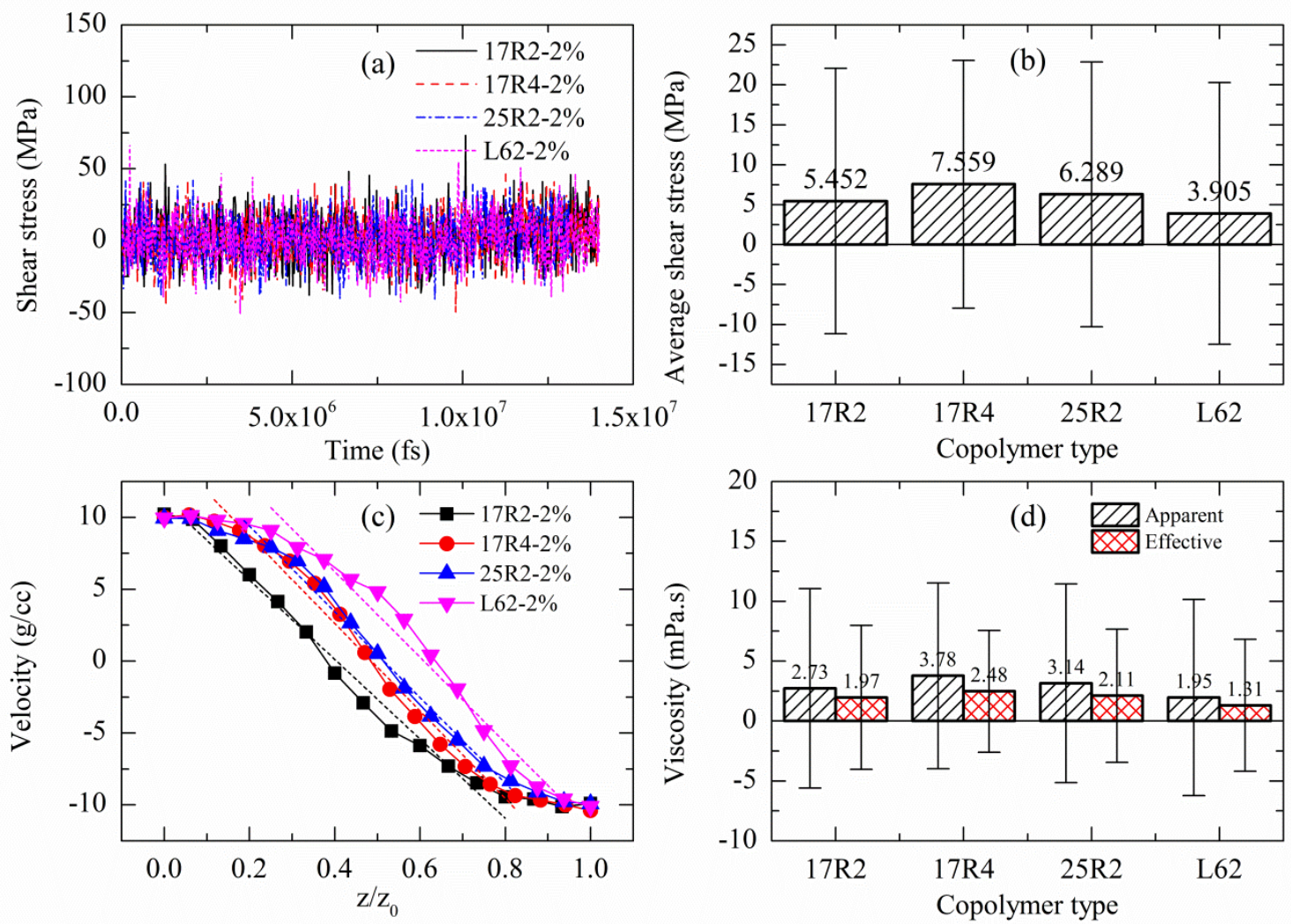

Fig. 18 (a) Time evolution of shear stresses; (b) average shear stresses; (c) velocity profiles across the film thickness and their fitted lines; and (d) apparent and effective shear viscosities for different triblock copolymer (16\%) lubricants

\section{DISCUSSION}

An interesting observation is that the DFTD results provide the larger adsorption energies of 1,2-DMP than 1,2-DME. However, the adsorption energies per PEO/PPO group for 25R2 and L62 without water in Fig. S5b in SM show a contrasting result. Moreover, these energies are lower than those obtained from the geometry optimization in DFTD. The molecular structure of copolymer presented in Fig. S3 provides the clues for our explanation. On one hand, the 'cloud' formation due to the aggregation of the bulk triblock copolymers in vacuum before it adsorbs onto the surface, has resulted in separated PEO and PPO regions. For instance, PEO occupies the first region of L62 that adsorbs onto the surface; it therefore has a larger adsorption energy/group than PPO. This cloud formation also reduces the adsorption energy of the copolymer as well as the adsorption energies per PEO/PPO group, as it prevents the outer molecules moving closer to the surface. On the other hand, the kinetic movement due to the influence of temperature causes the molecules to deviate from their optimal state (lowest energy) and reduces the interaction energy of the molecules. It is noted that the 'cloud' formation does not occur 
in the aqueous solution at low concentration. Therefore, PPO block has a larger adsorption energy than PEO, due to it stronger adhesion strength.

At the lubricant- $\mathrm{TiO}_{2}$ interface of aqueous solution, the copolymer concentration is almost contributed entirely by PPO block (Fig. 5-7, 13-16). These observations indicate that the $\mathrm{TiO}_{2}$ surface shows a hydrophobic property, which confirms previous experimental observation which indicates that $\mathrm{TiO}_{2}$ is considered as partially hydrophobic, with a water contact angle of $64.5^{\circ}$ [15].

The presence of water has reduced significantly the adsorption ability of copolymer. This could be explained by the amphiphilic property of PEO block that causes the dissolution of the copolymers into the water. The density profiles in Fig. 5-6, and 12-14 show that the copolymers form a layer with a significantly large peak of density at solid-fluid interfaces. This thick adsorption film thickness may work to reduce friction by reducing asperity contact. In this respect the copolymer that adsorbs on metal in monolayer is expected to protect the surface from friction without reducing the surface quality. As the copolymers adsorb physically onto rutile surface and this adsorption is reduced significantly in aqueous solution, they therefore could only work well in non-severe deformation condition [54].

In practice, 17R4 exists as one-phase unimer over wide concentrations and temperature ranges. It forms micelle at high concentration of $0.075 \mathrm{~g} / \mathrm{ml}$ within a narrow temperature range. 17R4 has more hydrophilic chains so it tends to be more soluble in water. The rheological results show that $17 \mathrm{R} 2$ and L62 with similar molecular weight and composition, but different structures have different tribological performance. The current result shown in Fig. 18 indicates that 17R4 has a higher viscosity than 17R2. This observation agrees with an experimental investigation by Taheri et al. [19].

For a surface coverage less than a monolayer, the total adsorption energy increases with molecular chain length. However, the results at higher concentration show that short-chain copolymer has higher adsorption energy than longer molecules, but the difference is insignificant. The adsorbed film thickness for monolayer depends on molecular structure in a propensity is that the higher is the ratio of PPO block the thicker is the adsorbed thin film. When the surface is fully covered by copolymer, a shorter molecular chain yields a higher peak of density profile or more densely adsorbed atoms on the surface than the longer chain (Fig. 13). This higher peak of density profile results in a larger adsorption energy for 17R2 compared with other R triblock copolymer. The largest desorbed amount of 17R4 copolymer from the surface during the shear process not only yields the lowest adsorption energy, but it also results in the highest shear stress as well as viscosity (Fig. 18). These facts indicate that the composition of copolymer influences both its molecular structure and rheological properties. 


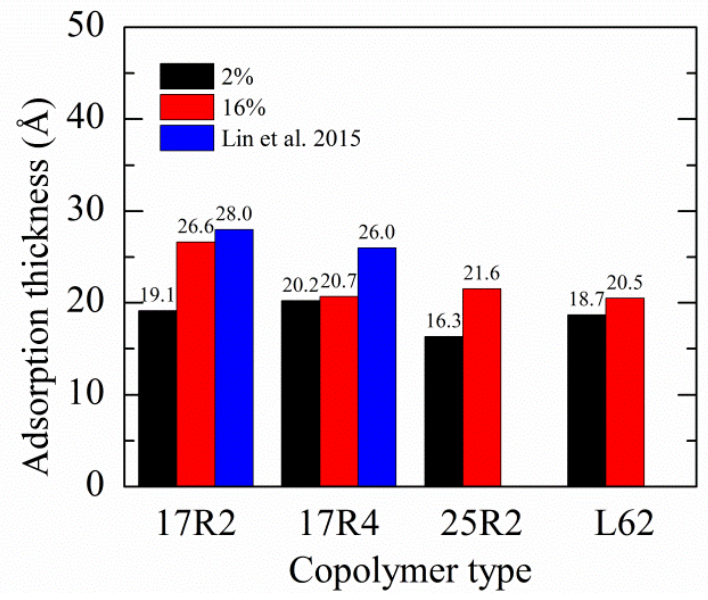

Fig. 19 Comparison of adsorption thickness of different Pluronics between the current MD simulations and previous experimental measurement by Lin et al. [15].

A comparison of adsorption thickness of different Pluronics between the current MD simulations and previous experimental measurements has been shown in Fig. 19 [15]. The adsorption thicknesses at high concentration (16\%) are higher than those obtained at low concentration (2\%). The discrepancy could be due to the influence of the number of adsorbed molecules. Besides, the thickness values obtained at high concentration could be more reliable than the lower one, as the larger number of molecules would give better statistical results. In fact, the adsorption thicknesses at high concentration are closer to the experimental investigation by Lin et al. using a horizontal TOF neutron reflectometer (SOFIA) at J-PARC/MLF [15].

There is a discrepancy between this theoretical calculation and experimental measurement of adsorption thickness. This discrepancy is caused by many reasons such as the surface condition which has been reported a roughness of $20 \AA$ for Ti coated surface [15], while an ideal smooth surface has been employed in current study. Additionally, the evaluated thickness of the anchored layer of PPO obtained from density profiles in Fig. 15, 23 is $\sim 8.5 \pm 0.8 \AA$. This thickness is larger than that of $5 \pm 1 \AA$ measured for 17R4, but it is lower than the thickness value of $18 \pm 1 \AA$ for $17 \mathrm{R} 2$ [15]. Despite some discrepancies, our simulated results still reveal an agreement with previous experiments. In fact, the obtained values at high concentrations (16\%) show a lower adsorption thickness for the case of higher weight ratio of PEO. Particularly, the thickness of the 17R4 lubricant film was the lowest compared with 17R2 and 25R2 films. This observation agrees well with Lin's results in which the 17R2 and 25R2 triblock copolymer with a longer length PPO chain forms a thicker film of lubricant on the hydrophobic surfaces [20].

The confined shear stretches some adsorbed copolymer molecules of 17R2 and 17R4 in the shearing direction (Fig. 13, 22). However, this effect is insignificant as there are still many molecules aligning 
in other directions (Fig. 8). The adsorption of copolymer onto the surface has resulted in a boundary condition where there is no slip at the solid-liquid interface. This boundary effect is clearer at a high concentration when the velocity profile shows a nonlinear gradient of velocity along the film thickness (Fig. 8). This nonlinear gradient has resulted in an increase of effective shear rate and a reduction of effective shear viscosity compared with the apparent ones. The increase of shear viscosity with copolymer concentration in mixed lubricant is due to the role of copolymer which has a higher viscosity than water. Additionally, although 17R2 and 17R4 have the same length chain of hydrophobic PPO block, the 17R4 lubricant, which contains a higher weight percentage of PEO, exhibited a higher viscosity than the 17R2 lubricant. This finding is consistent with previous experimental investigation [21]. For the same ratio of PEO, the 25R2 with a longer chain length results in a higher shear stress and shear-viscosity than 17R2. Additionally, the R Pluronics has a higher shear stress and viscosity than the L one.

\section{CONCLUSION}

The current work investigates the adsorption and rheology of triblock copolymer on $\mathrm{TiO}_{2}$ surface with and without water at atomic scale. An interfacial FF has been developed to describe interactions between the Pluronics and $\mathrm{TiO}_{2}$. The obtained results could be summarized as following:

i) There is a physisorption of Pluronics on $\mathrm{TiO}_{2}$ surface with adsorption energy for PPO block larger than PEO block. This adsorption energy is reduced significantly in the presence of water.

ii) PPO segments anchor onto $\mathrm{TiO}_{2}$ surface to form the anchor-buoy-anchor and buoyanchor-buoy-structures for R and L Pluronics, respectively, whilst the hydrophilic PEO segments extend away from the surface. The observed phenomenon is consistent with previous experimental investigations of triblock copolymer on $\mathrm{TiO}_{2}$ surface.

iii) The evaluated adsorption thicknesses agree qualitatively to the experimental investigations, and the copolymer with a higher weight ratio of PEO has a lower adsorption thickness than the lower one.

iv) The copolymers form a layer at solid-fluid interfaces with a significant large peak of density of PPO blocks. The adsorbed copolymer layer onto rutile surface results in an increase in effective shear rate of lubricant at high copolymer concentration, and the non-slip behavior has been observed in the shear. This nonlinear gradient has resulted in an increase of effective shear rate and a reduction of effective shear viscosity. The shear viscosity increases with copolymer concentration, and the copolymer with a higher weight ratio of PEO or chain length has a higher viscosity than the lower one. The L Pluronic has lower shear stress and viscosity than the R ones. 


\section{AUTHOR INFORMATION}

Corresponding Author: *E-mail: hongtao@uow.edu.au;

Present Addresses: School of Mechanical, Materials and Mechatronic Engineering, Faculty of Engineering and Information Sciences, University of Wollongong, Northfield Avenue, Wollongong, NSW, 2522, Australia

\section{ACKNOWLEDGEMENTS}

The authors would like to thank the National Computational Infrastructure (NCI) Australia for computing time on high-performance research computing environment. This project is supported by an Australian Research Council Discovery Projects DP130103973 and DP150103718.

\section{REFERENCES}

[1] Kosasih B, Novareza O, Tieu AK, Zhu H. Int J Surf Sci Eng. 2014;8:109-23.

[2] Laemmle JT. Aqueous metal-working lubricant containing polyoxypropylene-polyoxyethylenepolyoxypropylene block copolymers. United States Patent; 1984.

[3] Almgren M, Bahadur P, Jansson M, Li P, Brown W, Bahadur A. J Colloid Interface Sci. $1992 ; 151: 157-65$.

[4] Zhou Z, Chu B. Macromolecules. 1994;27:2025-33.

[5] Smith GD, Bedrov D, Borodin O. Phys Rev Lett. 2000;85:5583-6.

[6] Hammouda B, Ho D, Kline S. Macromolecules. 2002;35:8578-85.

[7] Calabro E, Magazu S. Advances in Physical Chemistry. 2013;2013:8.

[8] Bedrov D, Pekny M, Smith GD. J Phys Chem B. 1998;102:996-1001.

[9] Alexandridis P, Alan Hatton T. Colloids Surf, A. 1995;96:1-46. 
[10] Yang Z, Sharma R. Langmuir. 2001;17:6254-61.

[11] D'Errico G, Paduano L, Khan A. J Colloid Interface Sci. 2004;279:379-90.

[12] Lee S, Iten R, Müller M, Spencer ND. Macromolecules. 2004;37:8349-56.

[13] Sharma R, Bahadur P. Journal of Surfactants and Detergents. 2002;5:263-8.

[14] Li Y, Liu H, Song J, Rojas OJ, Hinestroza JP. ACS Appl Mater Interfaces. 2011;3:2349-57.

[15] Lin B, Zhu H, Tieu AK, Hirayama T, Kosasih B, Novareza O. Wear. 2015;332-333:1262-72.

[16] Jin J, Huang F, Hu Y, Jiang W, Ji X, Liang H, et al. Colloids Surf, B. 2014;123:892-9.

[17] Torcello-Gómez A, Wulff-Pérez M, Gálvez-Ruiz MJ, Martín-Rodríguez A, Cabrerizo-Vílchez M, Maldonado-Valderrama J. Adv Colloid Interface Sci. 2014;206:414-27.

[18] Liu X, Wu D, Turgman-Cohen S, Genzer J, Theyson TW, Rojas OJ. Langmuir. 2010;26:9565-74.

[19] Taheri R, Kosasih B, Zhu H, Tieu AK. Tribology Transactions. 2016:1-9.

[20] Lin B, Tieu AK, Zhu H, Kosasih B, Novareza O, Triani G. Wear. 2013;302:1010-6.

[21] Yang S, Yuan S, Zhang X, Yan Y. Colloids Surf, A. 2008;322:87-96.

[22] Liu H, Li Y, Krause WE, Pasquinelli MA, Rojas OJ. ACS Appl Mater Interfaces. 2012;4:87-95.

[23] Li B, Wong CH. Polymer. 2013;54:6008-18.

[24] Bedrov D, Smith GD, Chun B-W. Eur Polym J. 2010;46:2129-37.

[25] Hamaker HC. Physica. 1937;4:1058-72.

[26] Wong CH, Li B, Yu SK, Hua W, Zhou WD. Tribol Lett. 2011;43:89-99.

[27] Song X, Zhao S, Fang S, Ma Y, Duan M. Langmuir. 2016;32:11375-85. 
[28] Borodin O, Smith GD. J Phys Chem B. 2003;107:6801-12.

[29] Smith GD, Borodin O, Bedrov D. J Comput Chem. 2002;23:1480-8.

[30] Smith GD, Bedrov D. Macromolecules. 2002;35:5712-9.

[31] Smith GD, Borodin O, Bedrov D. J Phys Chem A. 1998;102:10318-23.

[32] Smith GD, Jaffe RL, Yoon DY. J Phys Chem. 1993;97:12752-9.

[33] Borodin O, Smith GD, Bandyopadhyaya R, Byutner O. Macromolecules. 2003;36:7873-83.

[34] Ta TD, Tieu AK, Zhu H, Zhu Q, Kosasih PB, Zhang J, et al. ACS Appl Mater Interfaces. 2016;8:5641-52.

[35] Kosasih B, Novareza O, Zhu H, Taheri R, Lin B, Tieu AK. Lubr Sci. 2016;28:299-315.

[36] McNamee CE, Higashitani K. Langmuir. 2013;29:5013-22.

[37] Ta TD, Tieu AK, Zhu H, Kosasih B, Zhu Q, Phan HT. Tribol Int. 2017;113:26-35.

[38] Ta DT, Tieu AK, Zhu HT, Kosasih B. J Chem Phys. 2015;143:164702.

[39] Harris RA, van der Walt H, Shumbula PM. J Mol Struct. 2013;1048:18-26.

[40] López-Lemus J, Chapela GA, Alejandre J. J Chem Phys. 2008;128:174703.

[41] Borodin O, Smith GD. J Phys Chem B. 2006;110:6279-92.

[42] Matsui M, Akaogi M. Mol Simul. 1991;6:239-44.

[43] Bandura AV, Kubicki JD. J Phys Chem B. 2003;107:11072-81.

[44] English NJ. Crystals. 2015;6.

[45] Ryckaert J-P, Ciccotti G, Berendsen HJC. J Comput Phys. 1977;23:327-41. 
[46] Hockney RW, Eastwood JW. Particle-Particle-Particle-Mesh (P3m) Algorithms. Computer Simulation Using Particles. Bristol, PA, USA: Taylor \& Francis; 1988. p. 267-304.

[47] Cramer CJ, Truhlar DG. Phys Chem Chem Phys. 2009;11:10757-816.

[48] Kim S-Y, van Duin ACT, Kubicki JD. J Mater Res. 2013;28:513-20.

[49] Kim S-Y, Kumar N, Persson P, Sofo J, van Duin ACT, Kubicki JD. Langmuir. 2013;29:7838-46.

[50] Audet C, J. E. Dennis J. SIAM Journal on Optimization. 2002;13:889-903.

[51] Ta TD, Tieu AK, Zhu H, Kosasih B. J Phys Chem C. 2015;119:12999-3010.

[52] Pritha D, Aditi S. Journal of Statistical Mechanics: Theory and Experiment. 2016;2016:083203.

[53] Novareza O. Tribology of aqueous copolymer lubricants for metal forming applications [Doctor of Philosophy thesis]. Wollongong: University of Wollongong; 2014.

[54] Ngaile G, Cochran J, Stark D. Proc Inst Mech Eng Pt B: J Eng Manuf. 2007;221:559-68. 